\title{
Allergen-encoding bone marrow transfer inactivates allergic $T$ cell responses, alleviating airway inflammation
}

\author{
Jane AL-Kouba, ${ }^{1}$ Andrew N. Wilkinson, ${ }^{1}$ Malcolm R. Starkey, ${ }^{2}$ Rajeev Rudraraju, ${ }^{1}$ \\ Rhiannon B. Werder, ${ }^{3}$ Xiao Liu, ${ }^{1}$ Soi-Cheng Law, ${ }^{1}$ Jay C. Horvat, ${ }^{2}$ Jeremy F. Brooks, ${ }^{1}$ Geoffrey R. Hill, ${ }^{4}$ \\ Janet M. Davies, ${ }^{5}$ Simon Phipps, ${ }^{3}$ Philip M. Hansbro, ${ }^{2}$ and Raymond J. Steptoe ${ }^{1}$ \\ 'The University of Queensland Diamantina Institute, University of Queensland, Brisbane, Australia. ${ }^{2}$ Hunter Medical \\ Research Institute and University of Newcastle, Newcastle, Australia. ${ }^{3}$ School of Biomedical Sciences, University of \\ Queensland, Brisbane, Australia. ${ }^{4}$ QIMR Berghofer Medical Research Institute, Brisbane, Australia. ${ }^{5}$ School of Medicine, \\ University of Queensland, Brisbane, Australia.
}

\begin{abstract}
Memory Th2 cell responses underlie the development and perpetuation of allergic diseases. Because these states result from immune dysregulation, established Th2 cell responses represent a significant challenge for conventional immunotherapies. New approaches that overcome the detrimental effects of immune dysregulation are required. We tested whether memory Th2 cell responses were silenced using a therapeutic approach where allergen expression in DCs is transferred to sensitized recipients using BM cells as a vector for therapeutic gene transfer. Development of allergen-specific Th2 responses and allergen-induced airway inflammation was blocked by expression of allergen in DCs. Adoptive transfer studies showed that Th2 responses were inactivated by a combination of deletion and induction of $T$ cell unresponsiveness. Transfer of BM encoding allergen expression targeted to DCs terminated, in an allergen-specific manner, Th2 responses in sensitized recipients. Importantly, when preexisting airway inflammation was present, there was effective silencing of Th2 cell responses, airway inflammation was alleviated, and airway hyperreactivity was reversed. The effectiveness of DC-targeted allergen expression to terminate established Th2 responses in sensitized animals indicates that exploiting cell-intrinsic $T$ cell tolerance pathways could lead to development of highly effective immunotherapies.
\end{abstract}

Conflict of interest: The authors have declared that no conflict of interest exists.

Submitted: November 30, 2015 Accepted: April 25, 2017 Published: June 2, 2017

Reference information: JCI Insight. 2017;2(11):e85742. https:// doi.org/10.1172/jci.insight.85742.

\section{Introduction}

Allergic diseases are perpetuated by dysregulated Th2 cell responses against environmental antigens. Cytokines (IL-4, IL-5, IL-9, IL-13, GM-CSF) produced by allergen-sensitized Th2 CD4+ $\mathrm{T}$ cells induce airway eosinophilia, chronic inflammation, and pathological changes such as mucous hypersecretion and bronchial smooth muscle hypereactivity, characteristic of atopic asthma. Allergen-specific IgE production is also promoted, and activation of mast cells and other FceR-bearing cells upon allergen encounter perpetuates inflammation and mediates acute airway constriction. In respiratory allergic disease, lower respiratory tract disease (e.g., asthma) is most tightly linked to pathogenic $\mathrm{T}$ cell responses, whereas upper respiratory tract disease (e.g., rhinitis) is strongly associated with IgE-mediated effects. In keeping with this, while anti-IgE treatments reduce exacerbations in asthma, therapies that address the effects of pathogenic $\mathrm{T}$ cells hold the greatest promise for asthma (1). Although, Th1/Th17 responses are implicated in neutrophilic asthma, allergic sensitization (atopy) is a key risk factor for all phenotypes (2) and underlies $\sim 75 \%$ of all cases, including chronic disease associated with allergen exposure and infection-induced exacerbations $(3,4)$. While a genetic component exists, allergic disease is apparent only after sensitization and effector/memory $\mathrm{T}$ cell differentiation has occurred. Therefore, therapies must contend with established memory and effector Th2 cell responses.

A cornerstone treatment for allergic disease is avoidance of known triggers and, for respiratory diseases, acutely or chronically administered short- or long-acting $\beta$-agonists and corticosteroids. While this may alleviate symptoms, it does not address the underlying immune pathology. Allergen-specific immunotherapies (SIT) are shown efficacious in meta-analyses $(5,6)$ but require years of treatment and are nonpermanent $(7$, 8). One potential approach to resolve this is development of newer, more rapid immunotherapies $(9,10)$. 
However, reinstatement of immune regulation by SIT may be difficult, as memory/effector Th2 cells may have diversified across a range of allergens, rendering Tregs unstable and subverting protective regulatory responses (11-13). Despite this, benefits are seen with SIT, indicating that efficacious immunotherapies would bring bona-fide medical and economic benefit $(14,15)$. In addition to dysregulated $\mathrm{Th} 2$ responses that trigger $\sim 50 \%$ of asthma, Th1/Th17-driven asthma is typically more severe $(16,17)$ and resistant to conventional treatments such as corticosteroids (18-20). Therapeutically, purging or silencing pathogenic allergenspecific effector and memory $\mathrm{T}$ cells as an alternative to approaches focussed on reinstating regulation may be advantageous. This would restore an environment devoid of pathogenic $\mathrm{T}$ cell specificities and potentially reverse immune-dysregulation both in Th2-biased allergies and possibly in the other more severe forms of asthma. This might be achieved by exploiting peripheral $\mathrm{T}$ cell tolerance, where the $\mathrm{T}$ cells are deleted or rendered unresponsive. Development of effective and rapid immunotherapies will be benefited by examining of a range of potential approaches from which common elements that underlie efficacy can be identified.

Antigen expression by antigen-presenting cells (APC) is robustly tolerogenic, overcomes genetically determined tolerance defects (21-23), and terminates memory and effector CD4 ${ }^{+}$and $\mathrm{CD} 8^{+} \mathrm{T}$ cell responses (24-26). Transfer of antigen-encoding BM or hematopoietic stem and progenitor cells (HSPC) using mild conditioning transfers a tolerogenic environment to hosts (27) and has therapeutic potential (28). Here, we sought to determine whether this could terminate pathogenic Th2 responses in an allergic airways disease $(\mathrm{AAD})$ setting. We demonstrate that antigen-encoding BM transfer ablated established Th2 cell responses to prevent allergen-elicited airway inflammation. Furthermore, established allergen-specific $\mathrm{T}$ cell responses were effectively alleviated, even when preexisting allergen-induced airway inflammation was present, and this alleviated airway inflammation and hyperresponsiveness.

\section{Results}

Allergen expression in DC prevents allergen-specific sensitization. Expression of antigens in APC, including $\mathrm{DCs}$, induces $\mathrm{T}$ cell tolerance and prevents antigen-specific $\mathrm{T}$ cell responses under classical Th1-inducing conditions $(23,27)$. To test this potential under Th2-inducing conditions, mice expressing OVA in DC (DC.OVA) and nontransgenic (non-Tg) controls were i.p. sham-treated (PBS/alum) or sensitized twice

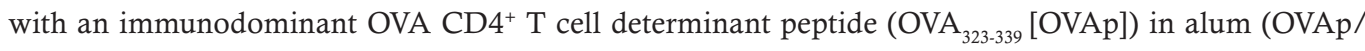
alum) (Supplemental Figure 1; supplemental material available online with this article; https://doi. org/10.1172/jci.insight.85742DS1). Spleen cells recovered from sensitized non-Tg mice produced substantial amounts of IL-5 and IL-13 in OVAp restimulated cultures (Figure 1, A and B). Mice expressing OVA (DC.OVA) did not show evidence of sensitization based on IL-5 or IL-13 production in restimulated cultures (Figure 1, A and B). Although present at low levels, IL-4 was significantly higher in supernatants from OVAp-sensitized non-Tg controls, compared with DC.OVA mice (Figure 1C). Notably, little IFN- $\gamma$ was produced (Figure 1D).

We next sensitized mice with a single i.p. administration of OVA protein/alum. OVA/alum sensitization also resulted in increased IL-5 and IL-13 production in OVAp-stimulated cultures from non-Tg mice but not DC.OVA mice (Figure 1, E and F). The frequency of IL-4-secreting cells detected in ELISpots (used since IL-4 is low in culture supernatants) was also substantially increased in OVA/alum-sensitized non-Tg but not DC.OVA mice (Figure 1G).

Allergen expression in DC prevents establishment of allergic airway inflammation. We next examined whether DC-expressed allergen prevented respiratory immune responses and Th2-mediated airway inflammation. DC.OVA and non-Tg controls were left unsensitized, sham sensitized, or OVAp/alum sensitized and exposed to OVA, administered i.n. They were then analyzed one day after the last i.n. challenge (Supplemental Figure 1). For mediastinal lymph node (MLN) that reflect the respiratory immune response to localized allergen challenge, OVAp restimulation elicited high levels of IL-5 and IL-13 and detectable levels of IL-4 in cultures from sensitized/i.n.-challenged non-Tg control mice (Figure 2, A-C). Cytokine production was much higher for MLN (Figure 2, A and B) than spleen (Figure 2, D and E), suggesting that i.n. challenge preferentially expanded OVA-specific Th2 responses in respiratory lymphoid tissues. Little IL-5, IL-13, or IL-4 was detected in supernatants from DC.OVA mice (Figure 2, A-E). When sensitized with OVA protein/ alum in place of OVAp/alum and challenged i.n. with OVAp, IL-4-producing cells detected by ELISpot were more frequent in MLN (Figure 2F) and spleen (Figure 2G) of sensitized/i.n.-challenged non-Tg mice than DC.OVA mice or sham-sensitized non-Tg controls. To determine whether accumulation of inflammatory cells was blocked, bronchoalveolar lavage fluid (BALF) was collected from allergen-challenged mice. 

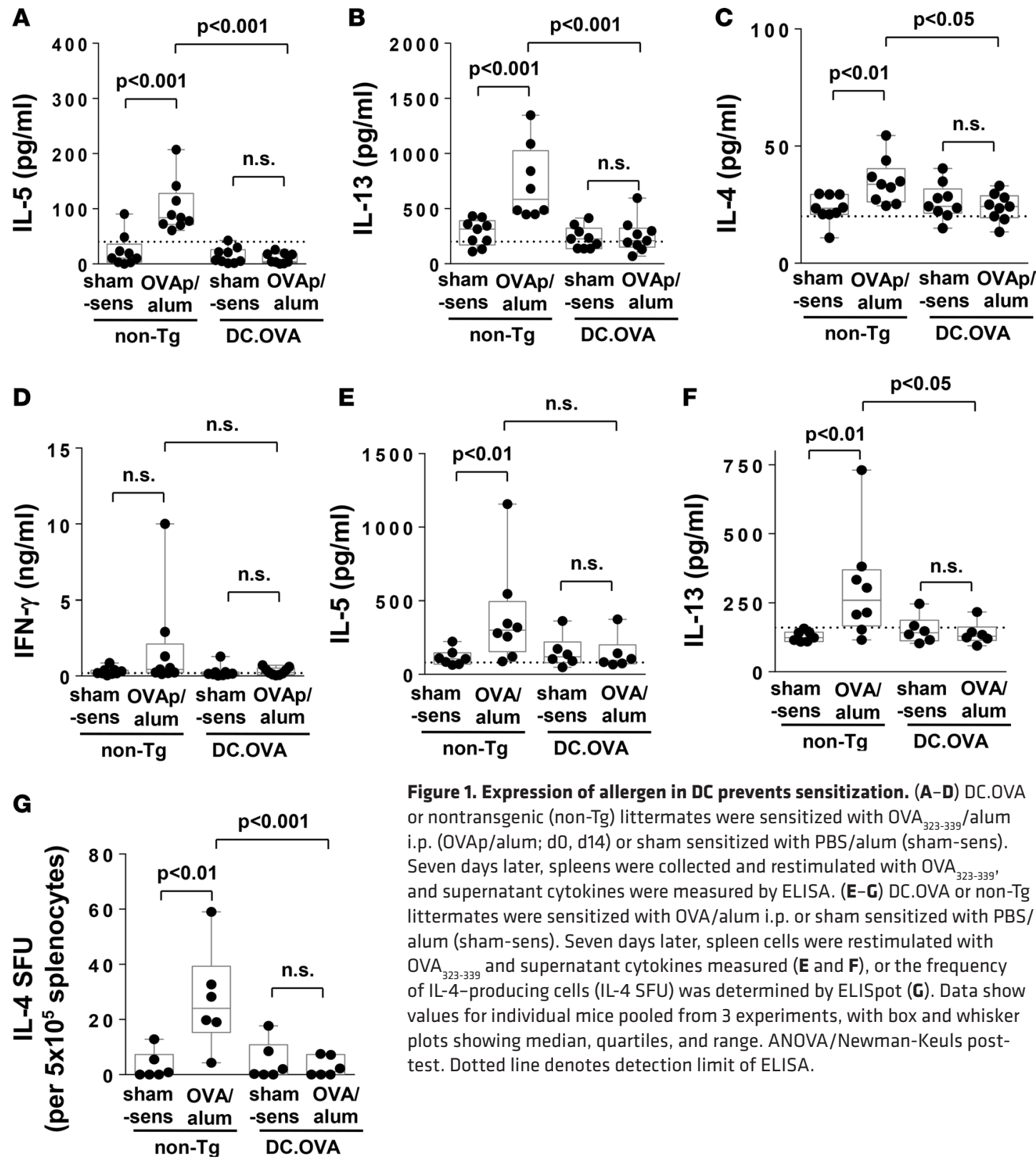

Figure 1. Expression of allergen in DC prevents sensitization. (A-D) DC.OVA or nontransgenic (non-Tg) littermates were sensitized with $\mathrm{OVA}_{323-339} /$ alum i.p. (OVAp/alum; d0, d14) or sham sensitized with PBS/alum (sham-sens) Seven days later, spleens were collected and restimulated with OVA $_{323-339}$ and supernatant cytokines were measured by ELISA. (E-G) DC.OVA or non-Tg littermates were sensitized with OVA/alum i.p. or sham sensitized with PBS/ alum (sham-sens). Seven days later, spleen cells were restimulated with $\mathrm{OVA}_{323-339}$ and supernatant cytokines measured (E and $\mathbf{F}$ ), or the frequency of IL-4-producing cells (IL-4 SFU) was determined by ELISpot (C). Data show values for individual mice pooled from 3 experiments, with box and whisker plots showing median, quartiles, and range. ANOVA/Newman-Keuls posttest. Dotted line denotes detection limit of ELISA.

Significantly higher numbers of eosinophils (Figure 2H) and other leukocytes (Supplemental Figure 2) were recovered in the BALF of sensitized/i.n.-challenged non-Tg mice compared with unsensitized or shamsensitized i.n.-challenged controls (Figure 2H). Expression of DC.OVA completely prevented allergen-elicited accumulation of $\mathrm{CD}^{+} \mathrm{T}$ cells (Supplemental Figure 2B) and eosinophils (Figure 2H).

Substantial peribronchiolar and perivascular infiltration was observed in H\&E-stained sections from sensitized/i.n.-challenged non-Tg mice (Figure 3A and Supplemental Figure 3). Semiquantitative scoring confirmed infiltration was significantly increased in sensitized/i.n.-challenged non-Tg mice compared with sham-sensitized/i.n.-challenged non-Tg mice (Figure 3B). Although a low-grade, nonspecific baseline infiltration was induced by i.n. challenge, infiltration was reduced in i.n.-challenged DC.OVA mice, regardless of whether they were sensitized or not compared with sensitized/i.n.-challenged non-Tg mice (Figure 3, A and B). Sensitization-specific infiltration after i.n challenge was completely blocked (Figure 3B). Similarly, no sensitization-associated goblet cell hyperplasia was detected in DC.OVA mice after i.n. challenge (Figure 3, C and D, and Supplemental Figure 3). Overall, expression of allergen in DC prevented the development or expansion of allergen-elicited $\mathrm{Th} 2$ responses systemically and in respiratory lymphoid tissues, and it blocked allergen-induced pulmonary inflammation. 


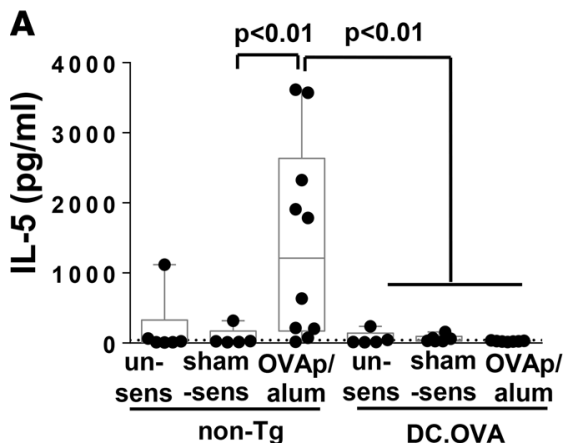

D

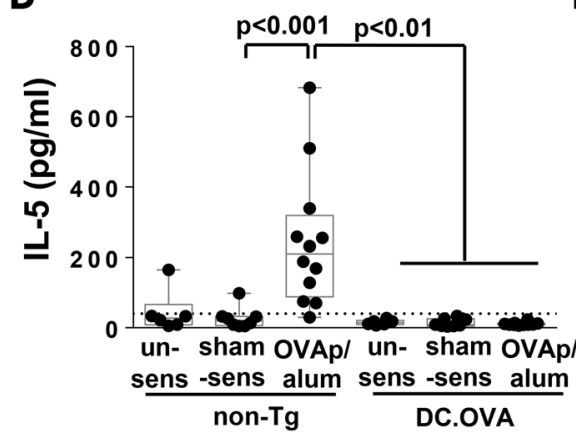

B

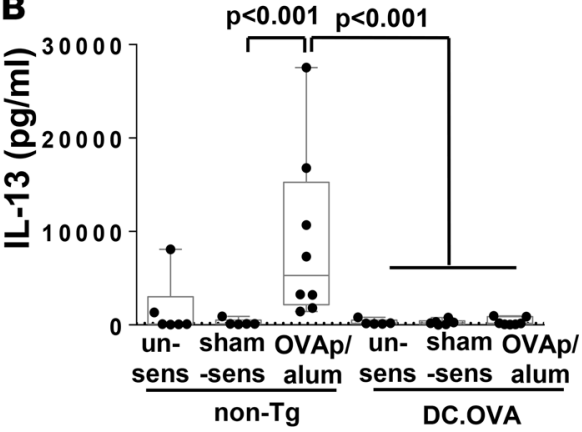

E

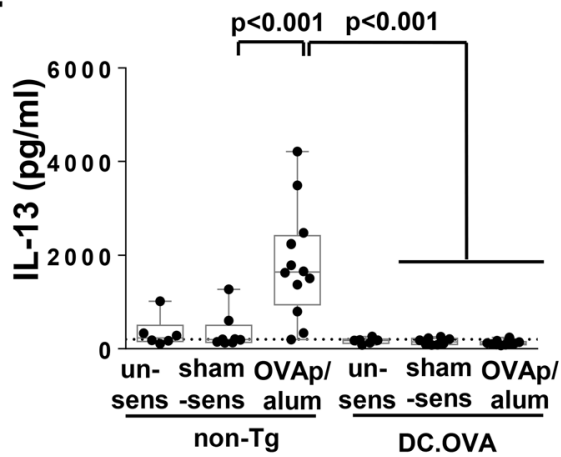

C

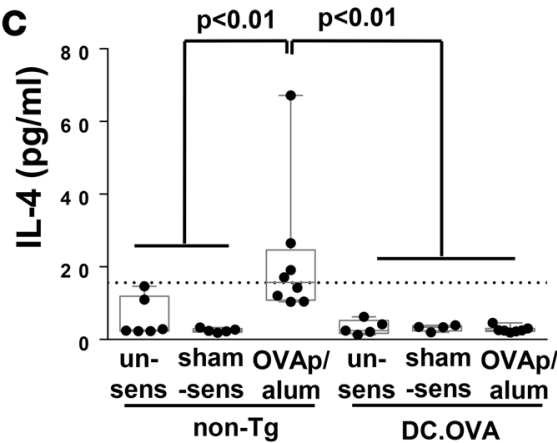

F

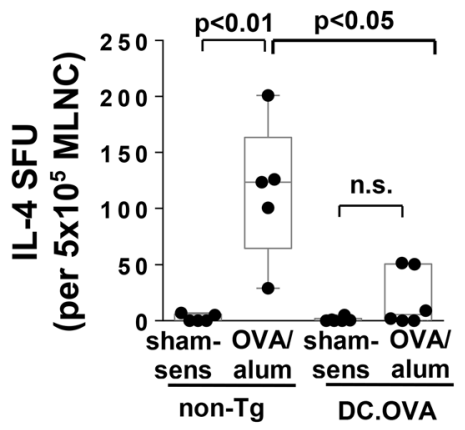

G

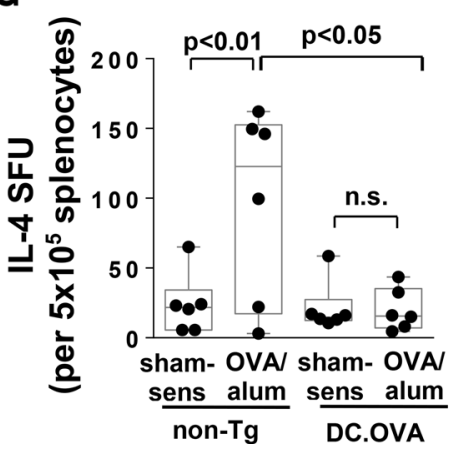

H

$p<0.01$

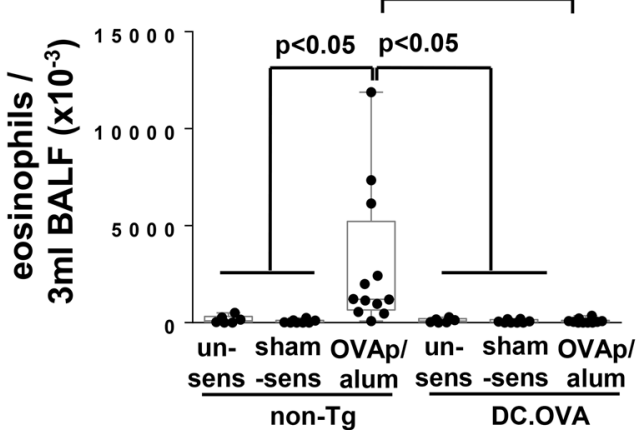

Figure 2. Expression of allergen in DC prevents airway inflammation. (A-E and H) DC.OVA or nontransgenic littermates (non-Tg) were sensitized with $\mathrm{OVA}_{323-339} /$ alum (OVAp/alum; d0, d14), were sham-sensitized with PBS/alum (sham-sens), or were left unsensitized (un-sens) and i.n. challenged with OVA daily (days 11-14 and 19-24 after sensitization). One day after the last i.n. challenge, mice were euthanized for analysis. Cytokine production in response to $\mathrm{OVA}_{323-339}$ restimulation of mediastinal lymph nodes (MLNC) (A-C) and spleen ( $\mathbf{D}$ and E) was determined by ELISA. Eosinophil infiltrate in bronchoalveolar lavage fluid (BALF) was determined by flow cytometry (H). (F and G) DC.OVA or non-Tg littermates were sensitized with OVA/alum (d0, d14) or sham-sensitized with PBS/alum (sham-sens) and i.n. challenged with OVA (days 11-14 and 19-24 after sensitization). One day after the last i.n. challenge, mice were euthanized for analysis. IL-4-secreting cells (IL-4 SFU) were enumerated in mediastinal lymph node cells (MLNC) (F) and spleen (C) by ELISpot. Data show values for individual mice pooled from 3-4 experiments, with box and whisker plots showing median, quartiles, and range. ANOVA/Newman-Keuls post-test. Dotted line denotes detection limit of ELISA.

Allergen expression in DC inactivates Th2-skewed effector/memory $C D 4^{+} T$ cells. Expression of antigen in DC inactivates Th1-skewed memory/effector $\mathrm{CD}^{+}$and $\mathrm{CD}^{+} \mathrm{T}$ cells $(24,26)$. To test whether this extends to $\mathrm{Th} 2$ skewed $\mathrm{CD}^{+} \mathrm{T}$ cells and to understand the mechanisms of tolerance induction in differentiated Th2-skewed memory $\mathrm{T}$ cells, we adoptively transferred OVA-specific Th2-skewed T cells to mice expressing DC.OVA. For these studies, we used OT-II T cell receptor (TCR) transgenic T cells that recognize OVAp in the context of I-A ${ }^{b}$, in conjunction with DC.OVA mice on a C57BL/6 background (DC.OVA [B6]) (23). We adapted previous protocols $(24,26)$ to generate $\mathrm{Th} 2$-skewed $\mathrm{T}$ cells for transfer. These $\mathrm{T}$ cells exhibited a CD44 ${ }^{\text {hi }} \mathrm{CD} 62 \mathrm{~L}^{\text {lo }}$ effector phenotype and were strongly $\mathrm{Th} 2$ skewed compared with cells differentiated under neutral nonskewing 
A

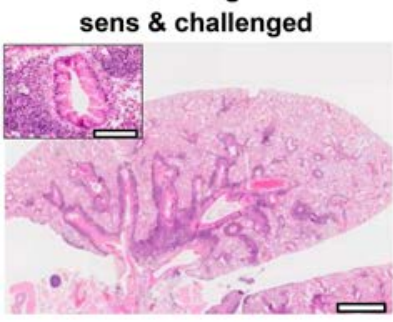

non-Tg sens \& challenged

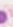

C

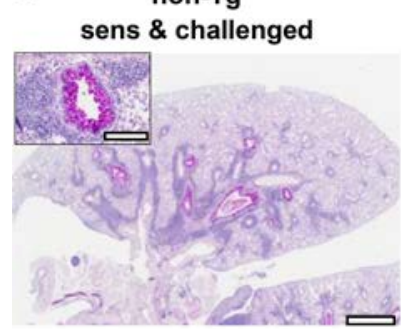

DC.OVA

sens \& challenged

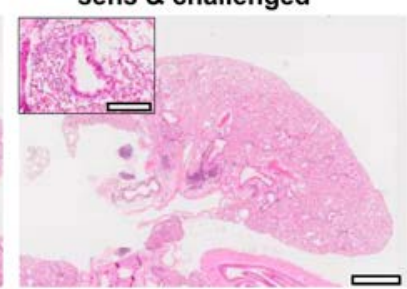

DC.OVA sens \& challenged

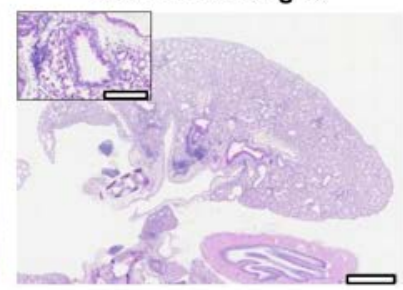

B

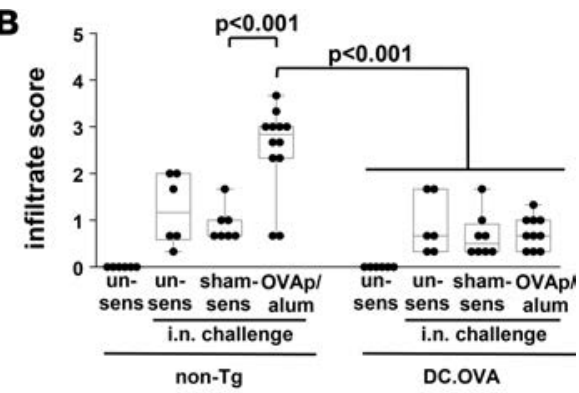

D

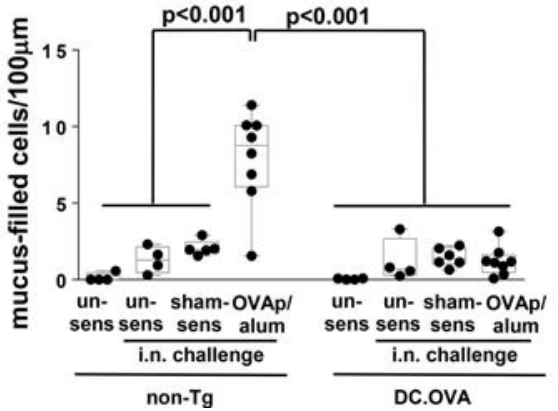

Figure 3. Expression of allergen in DC prevents pathology. DC.OVA or nontransgenic littermates (non-Tg) were sensitized with $\mathrm{OVA}_{323-339} / \mathrm{alum}$ (OVAp/alum; d0, d14), were sham-sensitized with PBS/alum (sham-sens), or were left unsensitized (un-sens) and i.n. challenged with OVA (days 11-14 and 19-24 after sensitization). One day after the last i.n. challenge, mice were euthanized for analysis. Lungs were collected and H\&E (A) or periodic acid-Schiff (PAS) (C) stained, and images were analyzed to define cellular infiltrate (B) and mucus-filled goblet cell frequency (cells per $100 \mu \mathrm{m}$ basement membrane) (D). Scale bars (A and C) depict $1 \mathrm{~mm}$ (low-power) and $200 \mu \mathrm{m}$ (high-power inset). Data are representative micrographs from 4-6 mice per group pooled from 3 experiments or show values for individual mice pooled from 3 experiments, with box and whisker plots showing median, quartiles, and range. ANOVA/Tukey's post-test

conditions (Supplemental Figure 4). When CFSE-labeled and transferred to DC.OVA (B6) mice, CFSE dilution indicated these cells had divided several times within 3 days of transfer (Figure 4A). In contrast, in non-Tg controls, few Th2 OT-II T cells showed evidence of division (Figure 4A). Transferred Th2 OT-II T cells rapidly accumulated in spleen and LN of DC.OVA (B6) mice, and their numbers increased substantially between 1 and 3 days after transfer (Figure 4, B and C). Following initial expansion, the OT-II population contracted and, within 3 weeks, the residual number of OT-II T cells was low (Figure 4, B and C). This is consistent with population expansion and deletion typically seen in adoptive transfer tolerance models $(23,24,26,29)$. To determine whether residual OT-II T cells were rendered unresponsive, recipients were challenged with OVA/ CFA (Supplemental Figure 1). A failure to respond here would indicate induction of tolerance. One week after challenge, substantial expansion of OT-II T cells had occurred in spleen and LN (pooled axillary, brachial, inguinal, mesenteric) of non-Tg recipients (Figure 4, D and E), indicating transferred cells had retained antigen responsiveness and were functional memory T cells. In contrast, in DC.OVA (B6) recipients, OT-II T cells did not expand after OVA/CFA challenge (Figure 4, D and E). Restimulation of spleen cell cultures with OVAp showed that OVA/CFA challenge elicited an increase in IL-5 and IL-13 production cells from recipients of Th2-skewed OT-II T cells but not DC.OVA (B6) recipients (Figure 4, F and G), indicating that residual OT-II $\mathrm{T}$ cells in DC.OVA (B6) mice were rendered functionally unresponsive. Similarly, when cells secreting IL-4 in response to OVAp were enumerated by ELISpot, OVAp-responsive IL-4-secreting cells were detected only in non-Tg recipients, and their frequency was increased by OVA/CFA challenge (Figure $4 \mathrm{H}$, OVAp no chall. vs. OVAp chall.). To determine whether classical IL-2-reversible anergy held OT-II T cell function in check in DC.OVA (B6) recipients, we also added IL-2 with or without OVAp to the ELISpot assay. IL-2 alone did not elicit IL-4 production, but addition with OVAp increased the frequency of IL-4-secreting cells detected relative to OVAp alone for OVA/CFA-challenged non-Tg Th2 OT-II recipients (Figure 4H). Addition of IL-2 did not restore IL-4 secretion by OT-II cells from DC.OVA (B6) recipients (Figure 4H). To gain further insights into the mechanisms of unresponsiveness, we analyzed the phenotype of residual OT-II T cells in non-Tg and DC.OVA (B6) recipients 21 days after transfer. We found that a substantial proportion of OT-II T cells in DC.OVA (B6), but not non-Tg recipients, expressed the coinhibitory molecules PD-1, LAG-3, and CD244, which are markers 
A
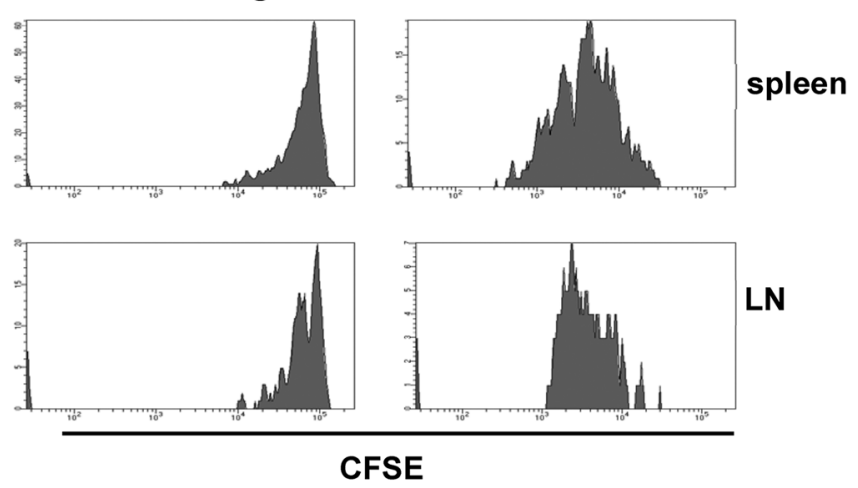

D

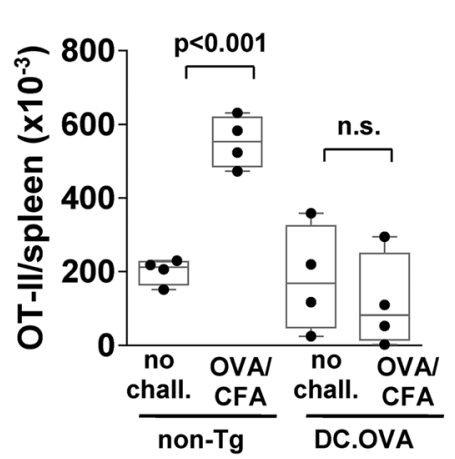

E

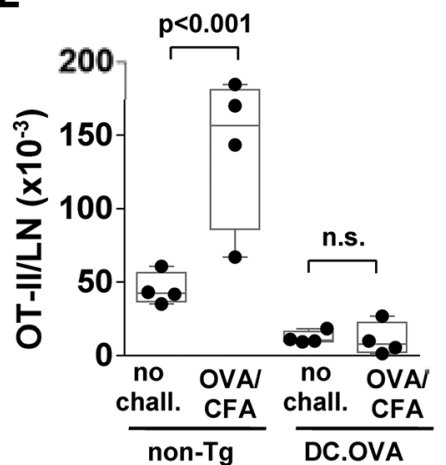

B

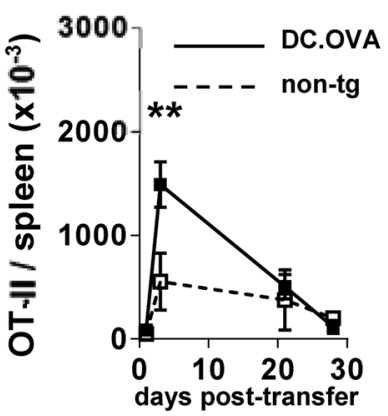

$\mathbf{F}$

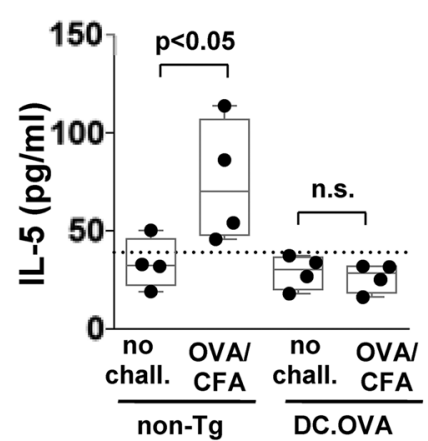

C

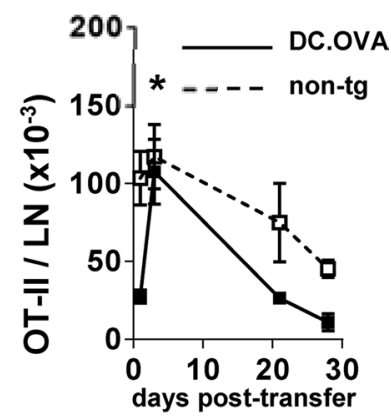

G

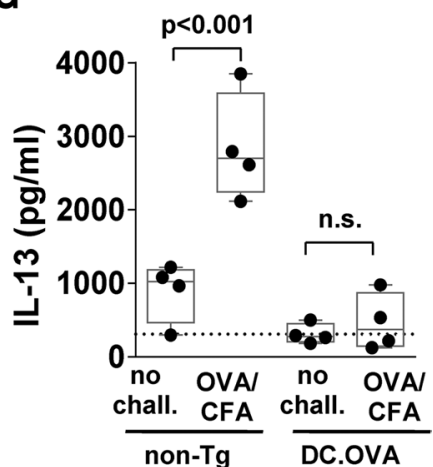

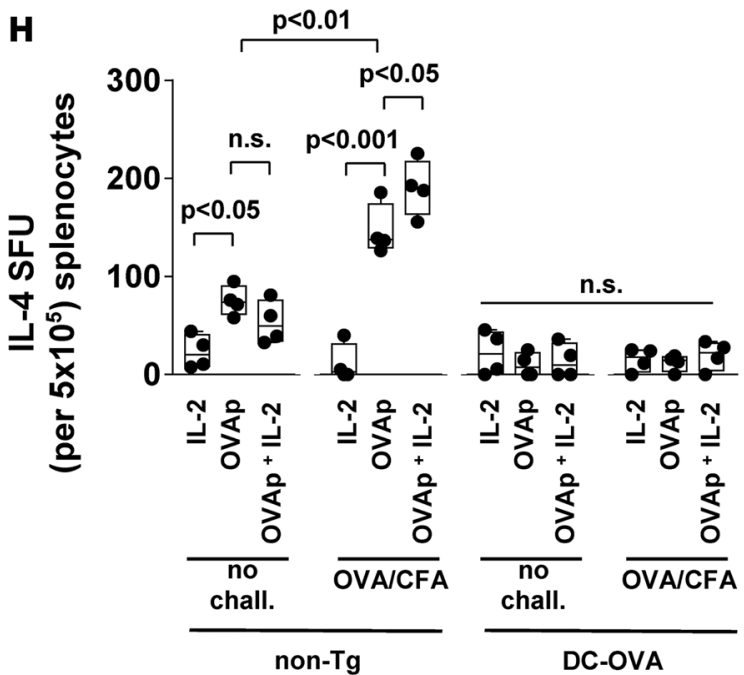
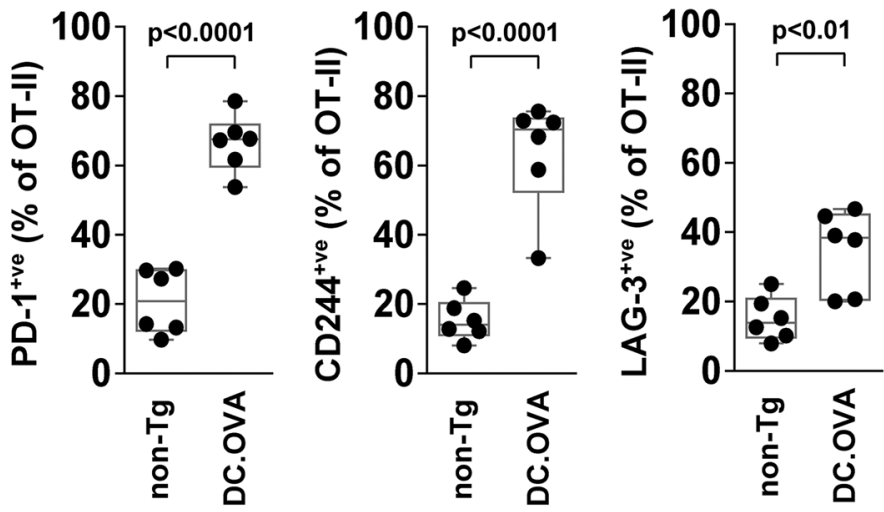

Figure 4. Expression of allergen in DC inactivates Th2 responses. Spleen and lymph node cells from CD45.1+ or CD45.2+ OT-II mice were cultured under Th2-inducing conditions and (A) cells were CFSE-labeled and transferred to congenically distinct non-Tg or DC.OVA (B6) mice. Three days later, spleens and lymph nodes (LN) were recovered for analysis. Data show CFSE intensity for transferred (CD45.1+CD4+) OT-II T cells. (B-H) Cells were transferred to congenically distinct non-Tg or DC.OVA mice, and at the indicated time points, tissues were recovered (B and $\mathbf{C})$, or 21 days later, mice were challenged (OVA/CFA) or not (no chall.) with OVA/CFA. A further 7 ( $\mathbf{D}$ and $\mathbf{E}$ ) days later, spleens and lymph nodes (LN) were collected, and OT-II T cells (CD45.1 $\left.1^{+} / \mathrm{CD} 4^{+}\right)$were enumerated using a cytometry-based counting assay. Five days after challenge (F-H), spleens were collected and cytokine production in response to $\mathrm{OVA}_{323-339}$ restimulation was measured by ELISA, or IL-4-secreting cells (IL-4 SFU) were enumerated by ELISpot. (I) Twenty-one days later, spleens were collected and OT-II T cells (CD45.2 $\left./ \mathrm{CD} 4^{+} / \mathrm{V} \alpha 2^{+}\right)$analyzed by flow cytometry. Data shown are (A) from a representative experiment of 2 with 2 mice per group, (B and C) mean \pm SD ( $n=4-6$ per group) pooled from 2 or 3 experiments. One asterisk denotes day 3 as significantly greater than day 1 , day $28(P<0.01)$, and day $21(P<0.05)$. Two asterisks denote day 3 as significantly greater than day 1 , day $28(P<$ $0.001)$, and day $21(P<0.01)$. (D-I) Values for individual mice pooled from 2 experiments, with box and whisker plots showing median, quartiles, and range. ANOVA/Tukey's post-test (B-H) or unpaired $t$ test (I). 

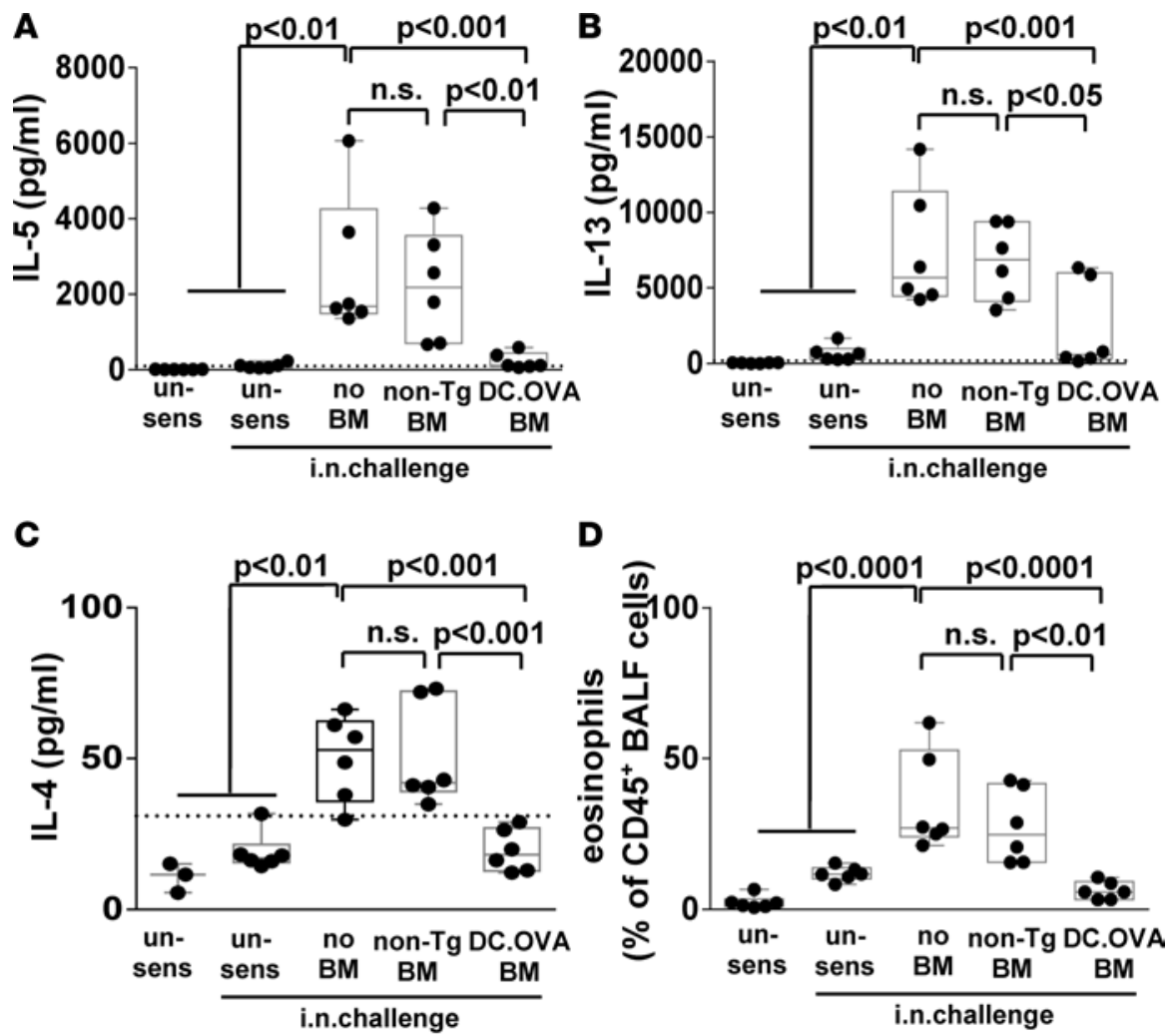

E sensitized,
non-Tg BM,
challenged
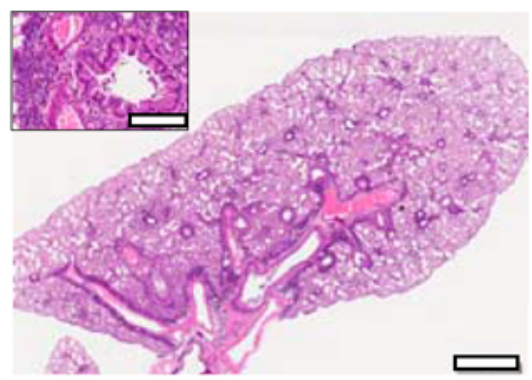

$\mathbf{F}$
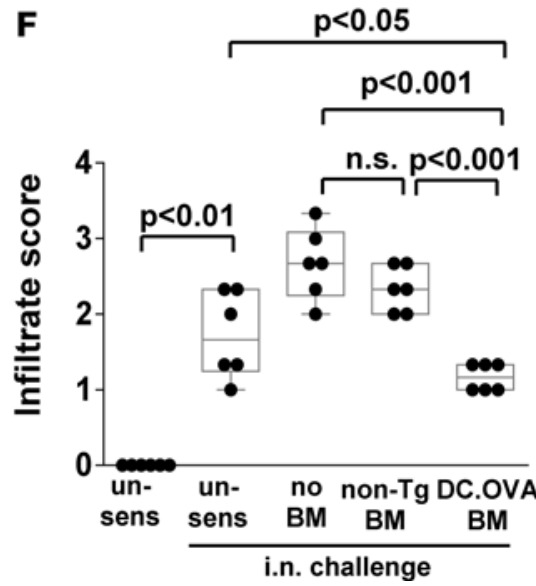

D

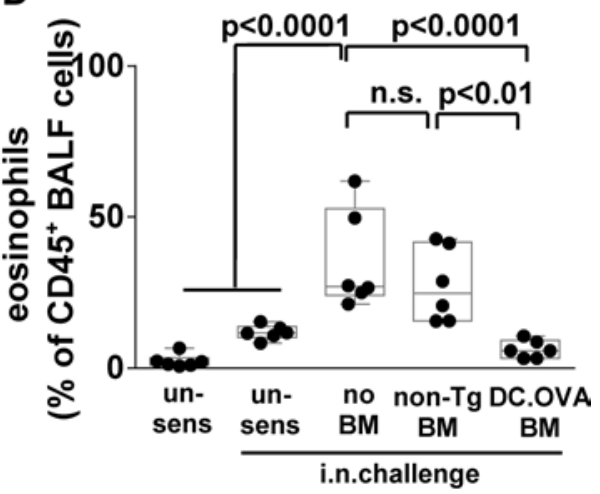

sensitized,

DC.OVA BM,

challenged

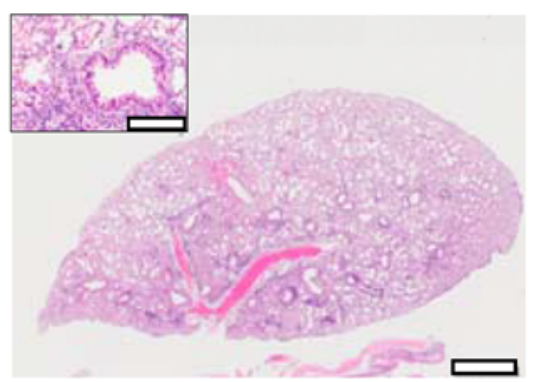

G

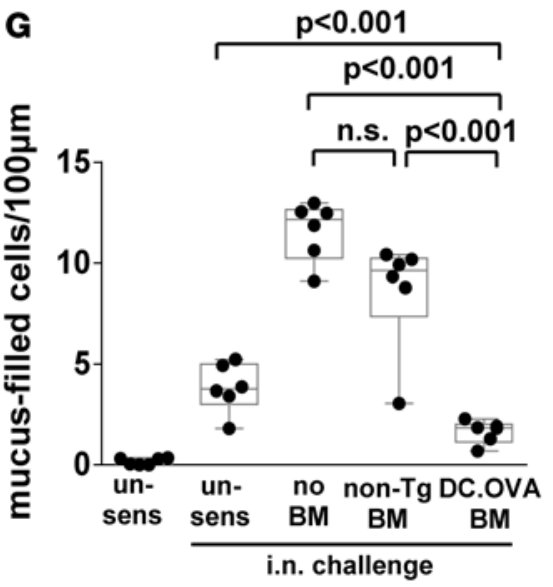

Figure 5. BM transfer terminates Th2 responses. (A-G) Nontransgenic BALB/c mice were sensitized with $\mathrm{OVA}_{323-339} /$ alum i.p. or sham-sensitized with PBS/alum (un-sens) twice, 14 days apart. One week after second sensitization, mice were irradiated (300 cGy) and DC.OVA or non-Tg BM transferred i.v. Four weeks after BMT, mice were i.n. challenged daily with OVA ( 5 consecutive days i.n., 4 days rest, 5 consecutive days i.n.). One day after the last i.n. challenge, mice were euthanized for analysis. Cytokine production in response to $\mathrm{OVA}_{323-339}$ restimulation of mediastinal lymph node cells (MLNC) (A-C) was determined by ELISA, and eosinophils in bronchoalveolar lavage fluid (BALF) was determined by flow cytometry (D). Lungs were collected and H\&E or periodic acid-Schiff (PAS) stained (E), and images were analyzed to define cellular infiltrate (F) and mucus-filled goblet cell frequency (G). Scale bars (E) depict $1 \mathrm{~mm}$ (low-power) and $200 \mu \mathrm{m}$ (high-power inset). Data are representative micrographs from 6 mice per group taken from 2 experiments or represent values for individual mice, with box and whisker plots showing median, quartiles, and range pooled from 2 independent experiments. ANOVA/Newman-Keuls post-test.

of T cell exhaustion (Figure 4I). Additionally, helios-expressing OT-II T cells were enriched in DC.OVA (B6) recipients $(12.1 \pm 3.5, n=$ 3) compared with non-Tg recipients $(3.5 \pm$ $1.5, n=3)$. No differentiation of transferred OT-II to Treg was apparent in either DC.OVA (B6) or non-Tg recipients (data not shown). Together, these data indicate a combination of deletion and induction of unresponsiveness, which most likely through exhaustion, underlie memory Th2 cell inactivation by DC-expressed allergen.

High levels of engraftment after transfer of BM and HSPC under immune-preserving nonmyeloablative conditioning. We next tested whether DC-expressed allergen would silence allergen-specific $\mathrm{T}$ cell responses in sensitized mice. Our approach was to transfer antigen-encoding BM to achieve de novo tolerogenic antigen expression in recipients (27). Importantly, this resembles the manner in which gene-engineered HSPC might be used clinically $(21,28)$, allowing us to perform proof-of-principle studies. Low-dose irradiation can serve as a mild conditioning procedure that enables high levels of donor BM engraftment and antigen expression in hosts, while preserving host immunity (27). When BALB/c mice were irradiated (300 cGy) and whole 

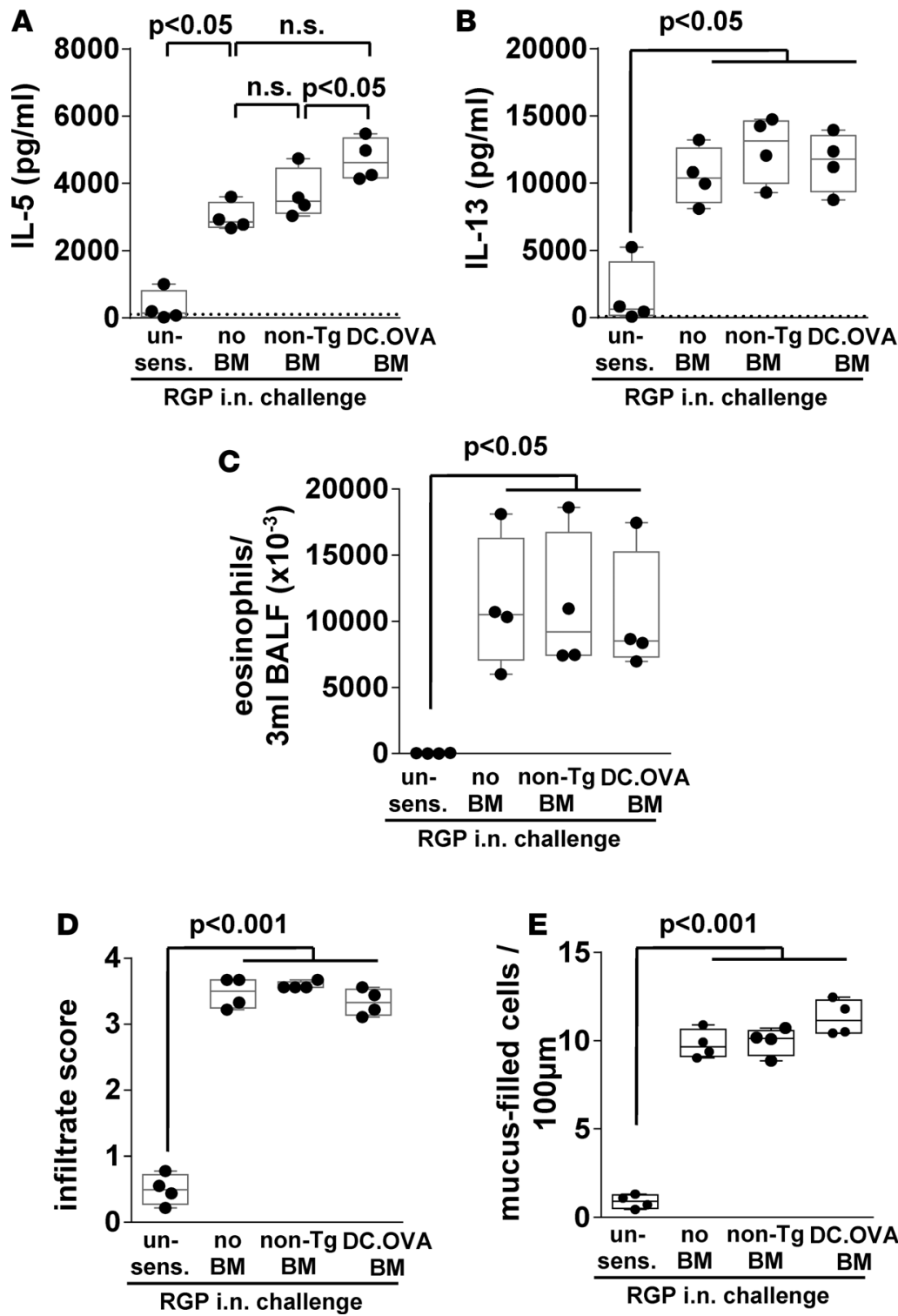
for 4 weeks and then i.n. OVA challenged to induce respiratory $\mathrm{Th} 2$ responses and test allergen elicitation of inflammation. Substantial IL-5 and IL-13 was produced, along with detectable levels of IL-4 in most OVAp-restimulated MLN cultures from control unirradiated, sensitized/i.n.-challenged (no BM) mice (Figure 5, A-C). Transfer of non-Tg BM had little effect on this (Figure 5, A-C), whereas OVA-encoding BM reduced IL-5 and IL-13 production to baseline and IL-4 to undetectable levels, equivalent to those in unsensitized controls, in the majority of mice (Figure 5, A-C).

Eosinophilia - which was prominent in BALF of unirradiated, sensitized/i.n.-challenged (no BMT) controls and non-Tg BM recipients - was reduced to baseline in DC.OVA BM recipients with levels equivalent to those in unsensitized, but i.n.-challenged, controls (Figure 5D). Therefore, allergen-encoding BM transfer effectively turned off preexisting and ongoing allergen-specific Th2 responses. Histology revealed that extensive perivascular and peribronchiolar infiltration (Figure 5, E and F, and Supplemental Figure 6) and goblet cell hyperplasia (Figure 5G and Supplemental Figure 6) evident in non-Tg BM recipients was significantly reduced, by comparison, in DC.OVA BM recipients. Inflammatory cell infiltration and goblet cell hyperplasia in DC.OVA BM recipients was reduced compared with unsensitized/challenged controls (Figure 5, F and G). This suggests that, as well as damping Th2 cell responses induced by the OVAp/alum sensitization and inflammation resulting from this, DC.OVA BM transfer may also dampen OVA-specific sensitization and inflammation developing from the i.n. delivery of OVA after BM transfer. 
A

$$
\begin{gathered}
\text { sensitized, challenged, } \\
\text { no BM } \\
\text { challenged }
\end{gathered}
$$

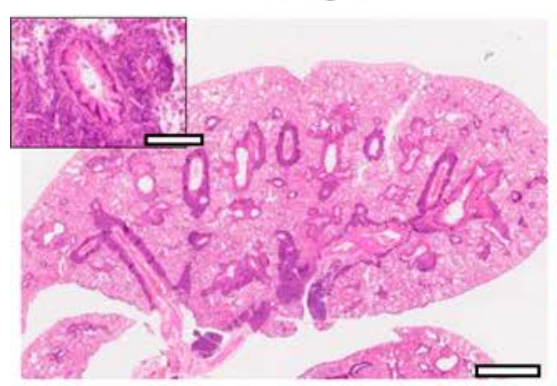

sensitized, challenged, DC.OVA BM challenged
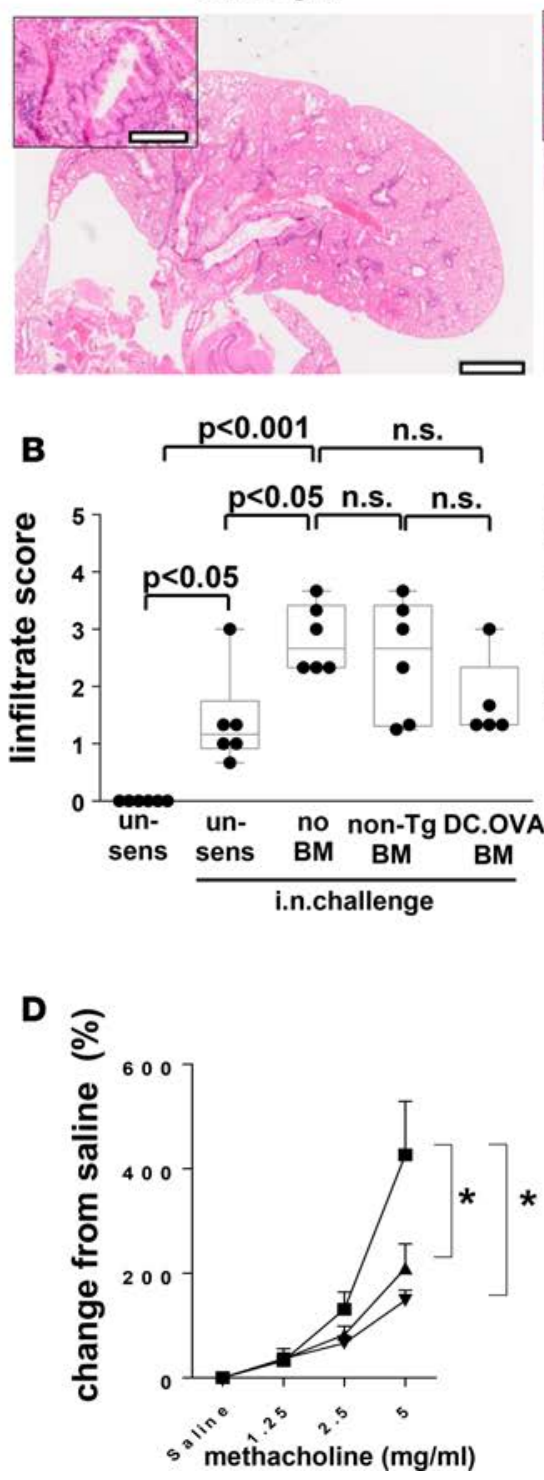

sensitized, challenged, non-Tg BM challenged

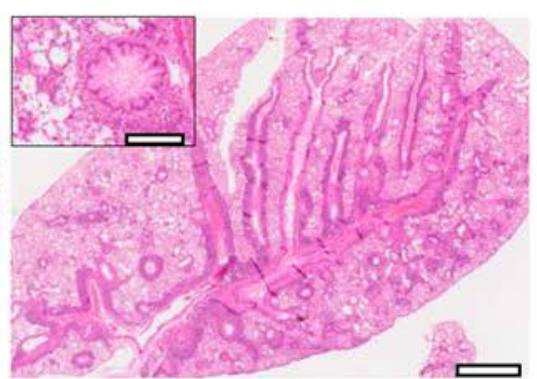

unsensitized, challenged, no BM challenged
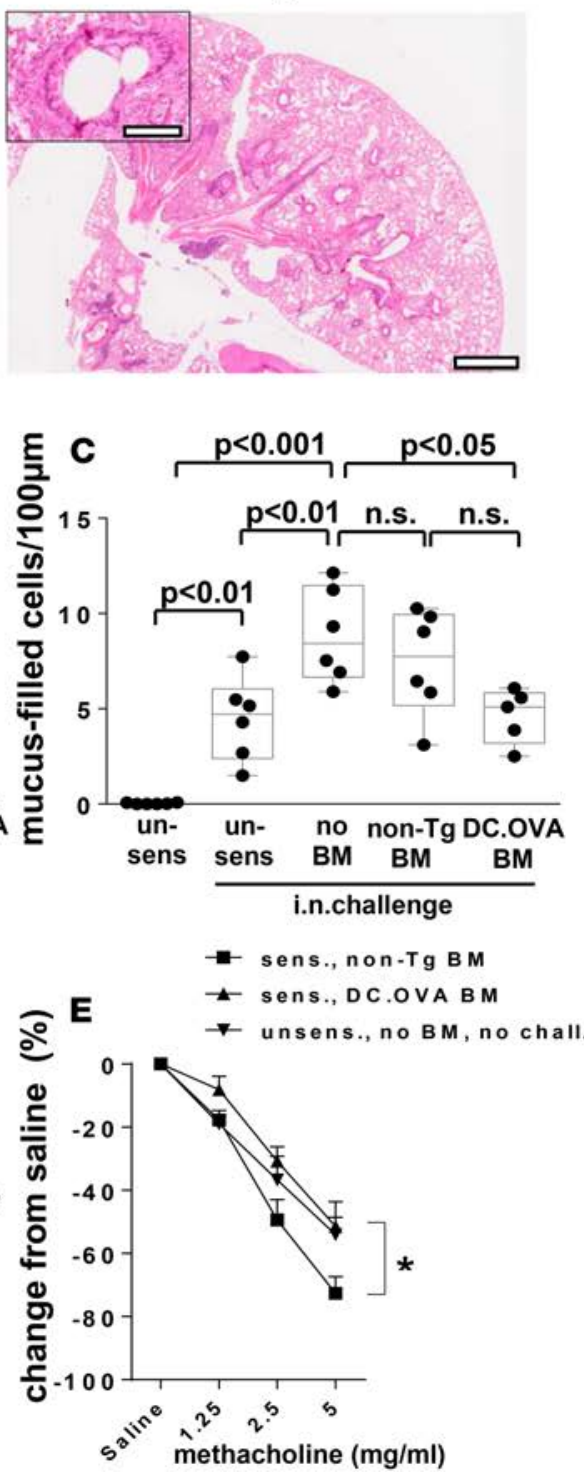

Figure 7. Transfer of OVA-encoding BM ameliorates established airway inflammation. (A-E) $\mathrm{BALB} / \mathrm{c}$ mice were sensitized twice with $\mathrm{OVA}_{323-339} \mathrm{I}$ alum (days 0,14 ) or not (un-sens) and challenged with OVA i.n. daily (days 11-14 and 19-24 after sensitization). One week later, some mice were irradiated (300 cGy) and injected with BM from DC.OVA (DC.OVA BM) or non-Tg (non-Tg BM) donors. Four weeks later, mice were i.n. challenged with OVA ( 5 days i.n., 4 days rest, 5 days i.n.). One day after the last i.n. challenge, mice were euthanized for analysis. (A-C) Untreated BALB/c mice (un-sens) were included for comparison. Lungs were collected and $\mathrm{H} \& E$ or periodic acid-Schiff (PAS) stained (A), and images were analyzed to define cellular infiltrate (B) and mucus-filled goblet cell frequency (cells/100 $\mu \mathrm{m}$ basement membrane) (C). Scale bars (A) depict $1 \mathrm{~mm}$ (low-power) and $200 \mu \mathrm{m}$ (high-power inset). ( $\mathbf{D}$ and $\mathbf{E}$ ) Mice were intubated, and changes in airways resistance and compliance in response to challenge with increasing doses of methacholine were measured. Data are representative micrographs of 6 mice per group from 2 experiments (A) or show values for individual mice pooled from 2 experiments, with box and whisker plots showing median, quartiles, and range (B and C) or mean \pm SD for 8 mice per group pooled from 2 experiments ( $\mathbf{D}$ and $\mathbf{E})$. ANOVA/Newman-Keuls post-test. ${ }^{*} P<0.05$.

Termination of Th2 responses by BM transfer is antigen specific. Modulation of Th2 responses and airway inflammation after transfer of DC.OVA BM but not non-Tg control BM implied that termination of OVA-specific memory Th2 cell responses was antigen specific. To formally test this, mice were concurrently sensitized with OVAp and rye grass pollen (RGP) extract, and then non-Tg or OVA-encoding DC.OVA $\mathrm{BM}$ were transferred and RGP responses were examined. Mice were i.n. challenged with RGP 4 weeks after BM transfer (Supplemental Figure 1). Neither transfer of nonTg BM nor DC.OVA BM inhibited IL-5 or IL-13 production elicited by RGP (Figure 6 , A and B). Similarly, eosinophil accumulation in BALF after i.n. RGP challenge was not significantly altered (Figure 6C), nor was the extent of inflammatory infiltrates (Figure 6D) or mucus hypersecretion (Figure $6 \mathrm{E}$ ), showing that - under conditions where OVA-specific responses were modulated - responses to an alternative antigen are preserved, indicating antigen-specificity.

Transfer of allergen-encoding BM after airway inflammation is established terminates allergen-specific Th2 responses and ameliorates pathology. Two key questions for development of immunotherapies remain open. Firstly, the question of whether modulation of $\mathrm{Th} 2$ responses is impaired once target organ inflammation is established, and secondly, the question of whether terminating allergen-specific Th2 responses 

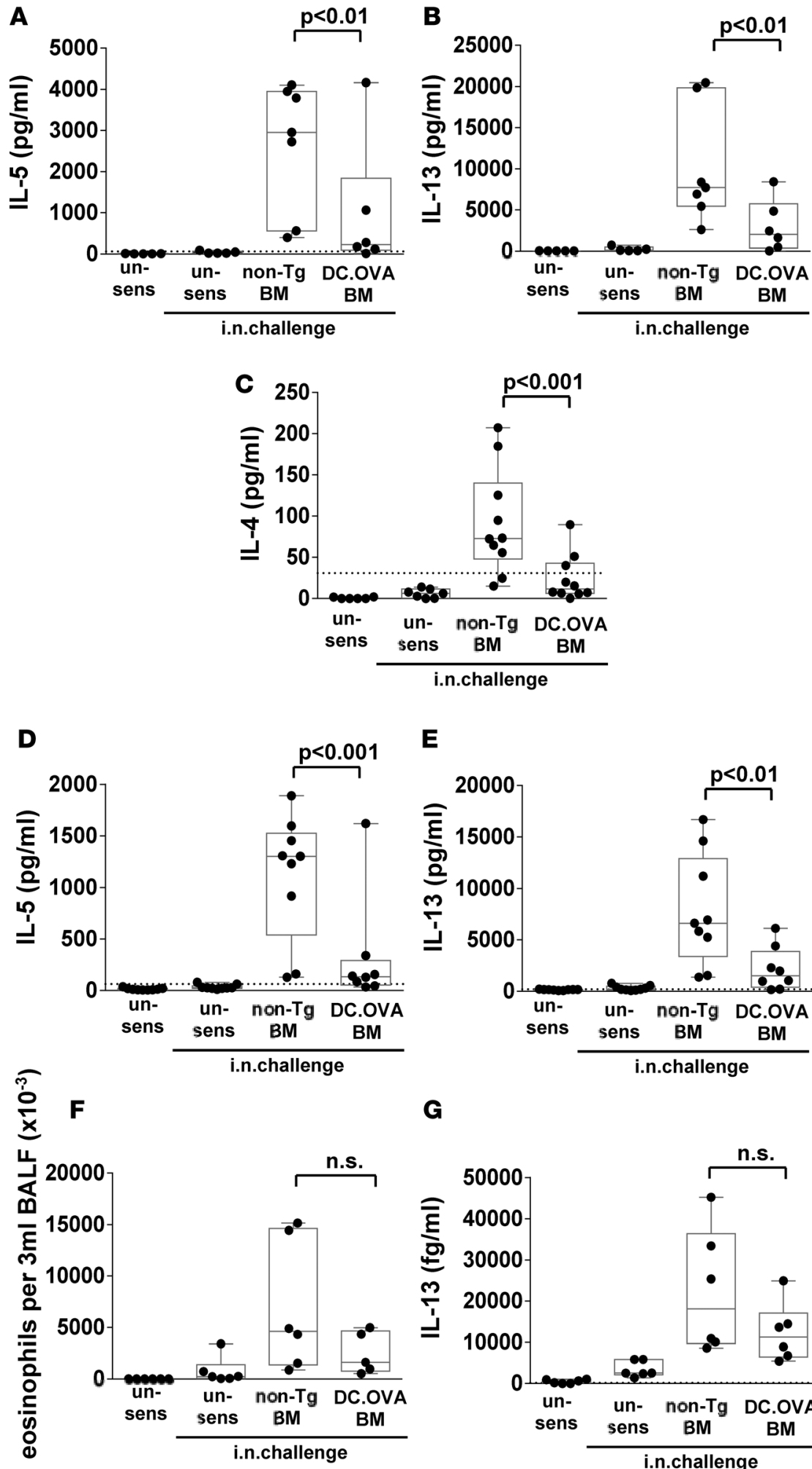

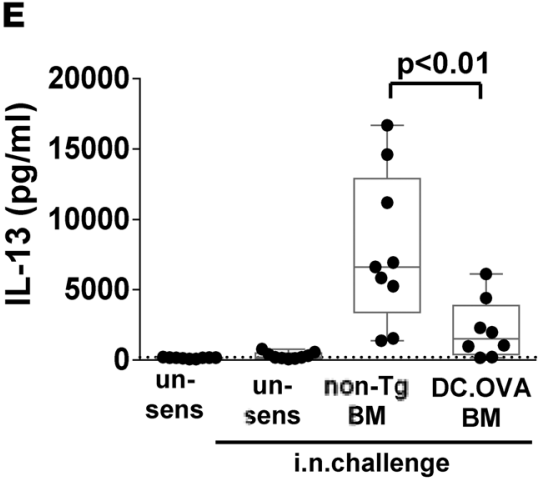

G

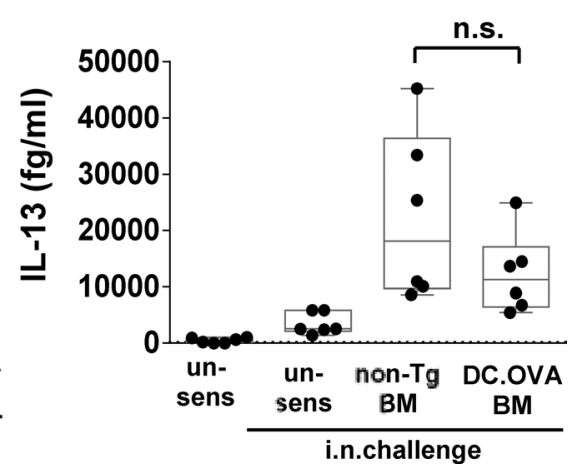

Figure 8. BM transfer terminates Th2 responses when inflammation is present. (A-C) BALB/c mice were sensitized with $\mathrm{OVA}_{323-339}$ /alum (days 0,14 ) and challenged with OVA i.n. daily (11-14 and 19-24 days after sensitization). One week later, some mice were irradiated (300 cGy) and injected with BM from DC.OVA (DC.OVA BM) or non-Tg (non-Tg BM) donors. Four weeks later, mice were i.n. challenged with OVA daily ( 5 days i.n., 4 days rest, 5 days i.n.). One day after the last i.n. challenge, mice were euthanized for analysis. Untreated BALB/c mice (un-sens) were included for comparison. Mediastinal lymph node cells $(\mathbf{A}-\mathbf{C})$ and spleen ( $\mathbf{D}$ and $\mathbf{E}$ ) were restimulated with $\mathrm{OVA}_{323-339}$, and cytokine production was determined by ELISA. Eosinophils in bronchoalveolar lavage fluid (BALF) were detected by flow cytometry (F). IL-13 in BALF was determined by CBA assay (C). Data show values for individual mice pooled from 3 or 4 experiments, with box and whisker plots showing median, quartiles, and range. Dotted line denotes ELISA detection limit. ANOVA/Newman-Keuls post-test.

alleviates inflammation. To explore this, BAL$\mathrm{B} / \mathrm{c}$ mice were sensitized i.p. and challenged i.n. with OVA to establish strong Th2 responses and airway inflammation prior to BM transfer (Supplemental Figure 1). After resting for 4 weeks, BM recipients, along with controls, were rechallenged i.n. (Supplemental Figure 1). Recipients of DC.OVA BM showed reduced periairway inflammatory infiltrates and goblet cell hyperplasia in the airways after i.n. challenge compared with sensitized/i.n.-challenged no BM transfer controls (Figure 7, A-C, and Supplemental Figure 7). Some residual cellular infiltration and goblet cells remained in recipients of DC.OVA BM, but this was largely similar to that in unsensitized/challenged controls (Figure 7, A-C, and Supplemental Figure 7). Recipients of non-Tg BM showed histological signs of pathology that were somewhat intermediate between no BM and DC.OVA BM groups, but they most closely resembled sensitized/i.n.-challenged no BM transfer controls. The extent of cellular infiltration and goblet cell hyperplasia in unsensitized/challenged mice compared with unsensitized/unchallenged controls suggests that i.n. challenge by itself leads to respiratory inflammation either through challenge-induced sensitization or nonspecific effects. In either case, inflammatory

effects were reduced to this baseline by transfer of OVA-encoding BM (Figure 7, B and C). We next tested whether BM transfer modulated physiological outcomes in terms of airways hyperesponsiveness (AHR; experimental outline shown in Supplemental Figure 1). Recipients of non-Tg BM showed a pronounced increase in airways resistance (Figure 7D) and reduction in lung compliance (Figure 7E) in response to increasing doses of methacholine. Notably, airways resistance and lung compliance for DC.OVA BM recipients did not differ from unsensitized/unchallenged mice that had no inflammation (Figure 7, B and 
C) and exhibit a normal baseline response. Therefore, while significant AHR remained in control BM recipients when airway inflammation existed prior to BM transfer, AHR was reduced or eliminated when allergen-encoding BM was transferred.

To understand whether reduced inflammation and AHR was attributable to reduced Th2 responses, MLN and spleen cultures were restimulated with OVAp. While substantial IL-5 and IL-13, and low but mostly detectable levels of IL-4, were present in MLN cultures (Figure 8, A-C) - or IL-5 and IL-13 in spleen cultures (Figure 8, D and E) - from non-Tg BM recipients, this was reduced in the majority of DC.OVA BM recipients. Interestingly, despite substantial reductions in Th2-associated cytokine production in MLN and spleen cultures, eosinophils and IL-13 in BALF were only moderately and not significantly reduced (Figure 8, F and G). On the whole, transfer of DC.OVA BM substantially terminated or inhibited many key pathological features of established $\mathrm{AAD}$ in the different settings tested.

\section{Discussion}

Development and maintenance of Th2 cell responses underlies a range of clinically important diseases. Often, loss of immune regulation occurs, which renders pathogenic $\mathrm{T}$ cell responses difficult to control using conventional immunotherapies or drugs (30). An alternative to conventional approaches is to exploit cell-intrinsic tolerance mechanisms to purge pathogenic $\mathrm{T}$ cell responses. Here, we demonstrate, firstly, that enforced expression of allergen in DC prevents allergen sensitization and terminates memory Th2 cell responses, and secondly, that established Th2 responses in sensitized recipients can be targeted and turned off by transfer of BM-encoding DC-targeted allergen expression. Furthermore, established Th2 responses can be terminated even in the presence of severe target-organ inflammation, a highly stringent test of the effectiveness of tolerogenic strategies. Under these conditions, terminating an allergen-specific $\mathrm{T}$ cell response relieves many features of end-stage, target-organ inflammation and AAD. Finally, this is achieved under immune-preserving conditions that would be essential for clinical application.

The mechanisms by which antigen-encoding BM transfer terminates established $\mathrm{Th} 2$ responses are yet to be defined in detail, but adoptive transfer studies here and elsewhere (24-26) indicate that a combination of activation-induced deletion and induction of unresponsiveness contribute. More recent studies (31) define a process of adaptive tolerance (32) that is similar to, but which may differ slightly from, classical $\mathrm{T}$ cell exhaustion (33) and underlies the unresponsive state induced in $\mathrm{CD}^{+}$memory $\mathrm{T}$ cells. Functional studies and the expression of a range of coinhibitory molecules in addition to helios, a marker of exhausted/self-tolerant $\mathrm{CD} 4^{+} \mathrm{T}$ cells $(34,35)$, indicate here that adaptive tolerance or exhaustion underlies maintenance of the unresponsive state in Th2-skewed OVA-specific CD4 ${ }^{+} \mathrm{T}$ cells. Our own studies indicate that continued expression of antigen is necessary for BM transfer to establish a long-term tolerogenic environment, implying the mechanisms involved in maintaining unresponsiveness of inactivated $\mathrm{T}$ cells could be exhaustion (33) or adaptive tolerance (32). Importantly, our observations here show that cell-intrinsic tolerance mechanisms induced by allergen-expressing DC proceed effectively in the face of established allergen-elicited inflammation. This suggests that exploiting cell-intrinsic tolerance mechanisms can overcome immune dysregulation and loss of Treg function associated with allergic sensitization. This is consistent with our previous findings that depletion of Treg (36) or NKT cells (R.J. Steptoe, unpublished observations) has little or no apparent effect on the capacity of DC to terminate $\mathrm{T}$ cell responses and that $\mathrm{T}$ cell unresponsiveness in this setting is not maintained by Treg. Our findings also indicate that helper functions elicited from, in this case, allergen-sensitized $\mathrm{CD}^{+} \mathrm{T}$ cells and allergen-driven inflammation, akin to previous reports, do not reverse DC tolerogenicity (23). This potentially overcomes the challenge for therapy of ongoing antigen exposure and inflammation and the need to rely on the reintroduction of regulation in an environment where this has failed due to genetic or environmental influences. In fact, the approach proposed here, which purges pathogenic specificities by removing ongoing pathogenic $\mathrm{T}$ cell responses while avoiding a strong requirement for functional Treg, could reinstate an environment where functional regulation can be restored.

As Th2 responses were effectively turned off, we determined whether this influenced established allergen-induced airway inflammation and pathology. Th2 cytokine production was reduced by DC.OVA BM transfer, and this had flow-on effects on pathology with the reduction of goblet cell hyperplasia and AHR. In humans, asthma symptoms result from smooth muscle hyperreactivity within small conducing airways, airway remodeling, and goblet cell hyperplasia; IL-13 is a key mediator of these processes in both mice and humans (37). This might imply that transfer of DC.OVA BM most rapidly modulates IL-13-mediated 
pathological processes $(1,38,39)$. That inflammatory cell infiltration in lung parenchyma and eosinophils in BALF were less affected might reflect a slower resolution or possibly development of Th2 independence of these processes. Further studies and analyses at later timepoints would help to clarify this. Whether inactivation of established allergen-specific $\mathrm{T}$ cell responses alters the responses of type 2 innate lymphoid cells (ILC2) in respiratory tissues and their potential to contribute to inflammation remains unclear, but this would be important to test in future studies. The presence of residual inflammatory infiltration may indicate the use of combination therapies where the addition of agents that reduce inflammation could lead to highly effective treatments. While not tested here, BM transfer may provide an innovative approach to the treatment refractory forms of asthma resulting from Th1- or Th17-biased responses. In either case, it is possible reversal of Th2 responses demonstrated here that reduced pathology in AAD could also relieve pathology in other allergic diseases.

Enforced expression of alloantigens, neoantigens, and allergens using genetic approaches, targeted to APC populations or not, is powerfully tolerogenic by the combined action of thymic and peripheral mechanisms (40) and prevents induction of $\mathrm{T}$ cell responses to expressed proteins/determinants $(23,41,42)$. Antigen-specific therapeutic approaches that aim to address established pathogenic antigen-specific $\mathrm{T}$ cell populations, be they naive or effector/memory, must exploit peripheral tolerance mechanisms. Antigen-expressing APC are able to mediate such peripheral tolerance effects $(24,25)$; however, this remains untested for allergen-induced airway inflammation. Where antigen-encoding BM or HSPC transfer is used with immune-preserving conditions, targeting expression of antigen(s) to differentiated APC is critical to prevent immune rejection of the transferred cells (27). Effective engraftment and establishment of hematopoiesis by the transferred HSPC is required for the continued genesis of antigen-expressing APC in order to generate a long-lived tolerogenic state in recipients. While, thymic mechanisms might prevent emergence of de novo antigen-specific T cells, the antigen-expressing APC that develop inactivate existing populations of antigen-specific T cells through peripheral tolerance mechanisms (27), and this has been demonstrated to extend to pathogenic $\mathrm{CD}^{+} \mathrm{T}$ cells (31). Whereas others have shown that expression of allergen by BM-derived cells can induce $\mathrm{T}$ cell and $\mathrm{B}$ cell tolerance in recipients of gene-engineered BM cells, this has been tested only under harsh, fully immunoablative conditions (43) where central tolerance mechanisms that influence development of naive lymphocyte populations may predominate. Furthermore, such studies have employed allergen constructs that are expressed ubiquitously and therefore transferred BM is subject to immune rejection (27). Here, we provide, to our knowledge, a novel finding that established that allergen-specific memory and effector populations are inactivated, while host immunity is largely preserved, and this is not prevented by active allergen-induced inflammation in target organs. Antibody and allergen-specific IgE-mediated effects were not tested here, as the present studies were intended purely to gain insight into effects on T cells. Expression of neoantigens and allergens using transcriptional targeting approaches is a powerful means to induce B cell tolerance $(44,45)$ in addition to T cell tolerance and can prevent sensitization and allergen-specific IgE production in response to experimental sensitization using model antigens (our unpublished data) or clinically important allergens (46). Mechanistic studies indicate that in double transgenic systems $(44,45)$ and in the polyclonal endogenous repertoire (47), B cell tolerance is mediated principally through cell-intrinsic central tolerance mechanisms, leading to antigen-specific B cell deletion during development and induction of $\mathrm{B}$ cell anergy when antigen is expressed in a membrane-bound form. Whether a component of $\mathrm{T}$ cell tolerance is required to limit T-dependent antibody responses is not yet clear, and whether mature B cells can be tolerized in the periphery independently of $\mathrm{T}$ cell help remains to be determined. However, depending on how antigen is delivered, for example in soluble form, inactivation of pathogenic Th2 responses may be crucial to controlling undesirable antibody responses. It is likely that HSC-based approaches, in addition to modulating Th2 responses, may be also effective for regulation of IgE responses. Certainly, transfer of transgenic or gene-engineered BM has the capacity to inhibit allergen-specific B cell responses and priming of antibody production, including IgE production (48-50), that is analogous to the effects on antigen-specific $\mathrm{T}$ cell responsiveness. Whether established B cell responses or persistence of allergen-specific IgE can be modulated as shown here for $\mathrm{T}$ cell responses remains to be determined, but some studies suggest that IgE-producing plasma cells may persist even in the absence of cognate T cell-specific signals (51). HSC-mediated gene therapy, as practiced currently, is not yet suitable for clinical application to mild diseases in young individuals (28), but we establish here the principle that DC-targeted approaches can alleviate unwanted Th2 responses and associated pathology. Advancements in stem cell-based approaches using either BM or personalized approaches, for instance employing induced pluripotent stem cells (iPSC), are likely to lead to 
more clinically attractive procedures. In the meantime, these findings provide insight into strategies for development of therapeutic approaches, including those not requiring antigen-encoding BM transfer.

Conventional antigen-specific immunotherapies applied for allergic diseases require sustained treatment for up to 3 years to achieve benefits lasting 5-10 years after treatment $(52,53)$, and compliance in a real-world setting is poor (54). These approaches attempt to restore immune regulation in environments where this has failed due to genetic or environmental influences. Here, we demonstrate an alternative approach that purges pathogenic memory and effector Th2 cells with high efficacy, limits allergen-induced inflammatory cell recruitment to the airway lumen, and ameliorates allergen-induced airways pathology. The silencing of Th2 immune responses and influences on inflammation are, as may be expected, more profound in the absence of preexisting airway pathology, but a substantial therapeutic effect is present that resets allergen-elicited inflammation and AHR to levels equivalent to controls. With further development for clinical application, this could provide a highly effective immunotherapy for established allergic diseases.

\section{Methods}

Mice. TCR transgenic DO11.10 and OT-II mice have been described $(55,56)$. To make CD45.1 ${ }^{+}$OT-II mice, OT-II mice were crossed with B6.SJL ${ }^{\text {ptrca }}$ mice. 11cOVA mice express a membrane-bound OVA construct under the control of the CD11c promoter, which targets OVA expression to CD11 ${ }^{\text {hi }}$ conventional DCs (23) and are referred to here as DC.OVA (B6) mice and were used as homozygous Tg/Tg. The DC.OVA

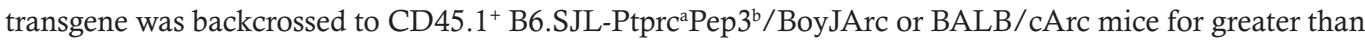
10 generations and were used as hemizygous $\mathrm{Tg} / \mathrm{O}$. Non- $\mathrm{Tg}$ controls were OVA ${ }^{\text {-ve }}$ littermate controls or BALB/cArc (for DC.OVA) or C57BL/6JArc mice (for DC.OVA [B6]). Male and female mice were used and aged 6-12 weeks at commencement of experiments. DO11.10, OT-II, 11c.OVA, CD45.1+11c.OVA and $11 \mathrm{c}$.OVA mice backcrossed to BALB/c were bred and maintained under specific pathogen-free conditions at Princess Alexandra Hospital Biological Resources Facility and Translational Research Institute Biological Resources Facility. BALB/c mice were purchased from Animal Resources Centre, Perth, Australia. Treated mice were shipped to Hunter Medical Research Institute for physiological testing. CD45.1 $1^{+}$ BALB/c (57) mice were housed at QIMR Berghofer and were a gift from Geoff Hill (QIMR Berghofer, Brisbane, Australia).

Immunizations and in vivo assays. For sensitizations, OVA (50 $\mu \mathrm{g}$, Sigma-Aldrich, Grade V), OVA ${ }_{323-339}$ (50 $\mu$ g, Auspep), or RGP (30 $\mu$ g) were mixed with $\mathrm{Al}_{2} \mathrm{O}_{3}$ gel (1 mg; Alu-Gel, Serva) in $200 \mu 10.9 \%$ saline and injected i.p. For i.n. challenge, OVA, OVA ${ }_{323-339}$, or RGP was diluted in pyrogen-free saline $(1 \mathrm{mg} /$ $\mathrm{ml}$ ), and $50 \mu 1$ was applied to the nares of lightly anesthetized (isoflurane) mice. For OVA/CFA challenge, equal portions of OVA $(1 \mathrm{mg} / \mathrm{ml})$ and CFA (Sigma-Aldrich) were emulsified, and $100 \mu 1$ was injected s.c. at the tail base. CFSE labeling of cells was as described (23). For bronchoalveolar lavage fluid $(3 \times 1 \mathrm{ml}$ mouse-tonicity PBS [MT-PBS]) was collected from $\mathrm{CO}_{2}$-euthanized mice. Cells were collected by centrifugation, and supernatants were frozen for storage. Airway hyperresponsiveness in terms of airway resistance and transpulmonary compliance in response to increasing doses of methacholine was performed as described $(58,59)$.

In vitro T cell activation and transfer. For in vitro generation of $\mathrm{CD}^{+}$effector $\mathrm{T}$ cells, lymph nodes (pooled brachial, axillary, inguinal, mesenteric) and spleens from CD $45.1^{+}$or CD $45.2^{+}$OT-II mice were prepared as described (26). For nonskewing, neutral conditions OVA $_{323-339}(10 \mu \mathrm{g} / \mathrm{ml})$ and $\mathrm{rhIL}-2(10 \mathrm{ng} / \mathrm{ml})$ were added. To skew toward a Th2 phenotype, rmIL-4 (10 ng/ml, PeproTech) and anti-IFN- $\gamma(5 \mu \mathrm{g} / \mathrm{ml}$, clone XMG1.2; BD Biosciences) were added in addition to OVA 323-339 and IL-2. After 4 days, cells were harvested, washed, and for adoptive transfer, $\mathrm{CD} 4^{+} \mathrm{T}$ cells were enriched using positive immunomagnetic enrichment (AutoMACS, Miltenyi Biotec). Cells were washed and $2 \times 10^{6}$ injected i.v. In some experiments, OT-II T cells $\left(\mathrm{CD} 45.2^{+}\right)$were transferred to CD45.1+ DC.OVA (B6) mice or littermate controls.

In vitro assays. In vitro restimulations were performed on spleen or LN single cell suspensions, or cells recovered from in vitro OT-II activation cultures were plated at $2 \times 10^{6} / \mathrm{ml}$ in complete RPMI with or without OVA $_{323-339}(10 \mu \mathrm{g} / \mathrm{ml})$. Culture supernatants were harvested after 72 hours, and ELISA was performed as described (26). ELISpots were performed as described (23) with or without additional added rhIL-2 (10 $\mathrm{ng} / \mathrm{ml}$, Peprotech). Intracellular cytokine staining was performed as described (24) using stimulation with PMA/ionomycin(3-4 hours).

BM transplantation. Donor mice were euthanized ( $\mathrm{CO}_{2}$ narcosis), and femurs and tibiae were collected. $\mathrm{BM}$ was flushed with MT-PBS/2.5\% FCS and dispersed into single cell suspensions, and erythrocytes were lysed 
with ACK buffer. Recipient mice were irradiated (300 cGy, ${ }^{137} \mathrm{Cs}$ source), and unfractionated $\mathrm{BM}\left(2 \times 10^{7}\right.$ cells) or FACS-sorted lin ${ }^{\text {-ve }} \mathrm{c}$-kit ${ }^{+v e}$ hematopoietic progenitor cells $\left(\mathrm{HPC} ; 1 \times 10^{5}\right)$ were injected i.v. as described (27).

Histological analysis. Serial sections (at $3 \times 500 \mu \mathrm{m}$ intervals) were prepared from formalin-fixed, paraffin-embedded tissue. Sections were stained with H\&E or with periodic acid-Schiff (PAS) reagent and scanned at 20× magnification with an Olympus VS120 (Olympus Corporation). Images were prepared using the OlyVIA2.6 software (Olympus Soft Imaging Solutions GmbH). Mucus-filled (PAS ${ }^{+}$) cells/100 $\mu \mathrm{m}$ airway epithelium (58) were enumerated in a blinded manner using NIS Elements Basic Research Software (BR 3.264 bit, Nikon) or Visiopharm. Data were collated in Microsoft Excel, unblinded, and means were calculated for each mouse. Means for each lobe and each mouse were then calculated. For inflammatory infiltrate analysis, a semiquantitative scoring system was used similar to that described previously (58) with the following scoring parameters: 0 , no infiltrate in parenchyma or surrounding airways; 1 , sparse $(0 \%-20 \%$ airways and vessels with infiltrate $<50 \%$ of circumference) $; 2$, moderate $(0 \%-20 \%$ airways and vessels with infiltrate $>50 \%$ of circumference); 3 , extensive $(20 \%-70 \%$ of large airways surrounded by cellular infiltrate); 4 , massive (70\%-100\% of large airways and small airways surrounded by infiltrate, areas of infiltrate away from airways/vessels). The 3 individual lobes for each mouse lung was scored in a blinded fashion and the average plotted.

Flow cytometry. Fluorochrome-conjugated antibodies were purchased from BioLegend or BD Pharmingen and are listed in Supplemental Table 1. Staining and cytometry was as described (23). Data were collected using FACSCalibur, FACSCanto, LSRII, Fortessa X-20 (BD Biosciences), or Gallios (Beckman Coulter) cytometers and analyzed with CellQuest, FACSDiva, Kaluza, or Flow-Jo software. To enumerate cell number, cytometric bead-based counting assays were performed as described (23).

Statistics. One-way ANOVA with Newman-Keuls or Tukey's post-test for multiple comparisons; 2-tailed, paired $t$ test was used for matched pairwise analyses or unpaired $t$ test used for unmatched pairwise sample comparison (GraphPad Prism 5 or Prism 6). A $P$ value less than 0.05 was considered significant.

Study approval. Experiments were approved by The University of Queensland Animal Ethics Committee or the University of Newcastle Animal Ethics Committee.

\section{Author Contributions}

JAK, ANW, RR, MRS, and JCH designed and performed experiments and analyzed data; XL, SCL, RNW, and JFB performed experiments; GRH provided reagents; and JAK, JMD, SP, PMH, and RJS wrote the manuscript.

\section{Acknowledgments}

This work was supported by NHMRC Project grant GNT1013066 (RJS, JMD) and Asthma Foundation of Queensland (RJS). RJS was a recipient of Australian Research Council Future Fellowship (FT110100372). MRS was a recipient of an NHMRC Early Career Fellowship (GNT1073317). PMH was a recipient of an NHMRC Principal Research Fellowship (GNT1079187) and a Brawn Research Fellowship. SP was a recipient of Australian Research Council Future Fellowship (FT130100518). GRH is an Australian National Health and Medical Research Council senior principal research fellow. The authors acknowledge the technical contribution of Tracy Doan, Chantelle Reid, and Ryan Galea and members of TRI Flow Cytometry, Microscopy and Biological Resource Facilities to experimental studies.

Address correspondence to: Raymond J. Steptoe, UQ Diamantina Institute, Translational Research Institute, 37 Kent Street, Woolloongabba, 4102 Queensland, Australia. Phone: 61.7.3443.6959; E-mail: r.steptoe@uq.edu.au.

JMD's present address is: School of Biomedical Science, Queensland University of Technology, Brisbane, Australia.

1. Hansbro PM, et al. Th2 cytokine antagonists: potential treatments for severe asthma. Expert Opin Investig Drugs. 2013;22 (1):49-69.

2. Holt PG, Strickland DH, Sly PD. Virus infection and allergy in the development of asthma: what is the connection? Curr Opin Allergy Clin Immunol. 2012;12 (2):151-157.

3. Asher MI, et al. Worldwide time trends in the prevalence of symptoms of asthma, allergic rhinoconjunctivitis, and eczema in childhood: ISAAC Phases One and Three repeat multicountry cross-sectional surveys. Lancet. 2006;368 (9537):733-743. 
4. Hansbro NG, Horvat JC, Wark PA, Hansbro PM. Understanding the mechanisms of viral induced asthma: new therapeutic directions. Pharmacol Ther. 2008;117 (3):313-353.

5. Calderón MA, Casale TB, Togias A, Bousquet J, Durham SR, Demoly P. Allergen-specific immunotherapy for respiratory allergies: from meta-analysis to registration and beyond. J Allergy Clin Immunol. 2011;127 (1):30-38.

6. Di Bona D, Plaia A, Leto-Barone MS, La Piana S, Di Lorenzo G. Efficacy of subcutaneous and sublingual immunotherapy with grass allergens for seasonal allergic rhinitis: a meta-analysis-based comparison. J Allergy Clin Immunol. 2012;130 (5):1097-1107.e2.

7. Sandrini A, Rolland JM, O'Hehir RE. Current developments for improving efficacy of allergy vaccines. Expert Rev Vaccines. 2015;14 (8):1073-1087.

8. Calderon M, Mösges R, Hellmich M, Demoly P. Towards evidence-based medicine in specific grass pollen immunotherapy. Allergy. 2010;65 (4):420-434.

9. Jutel M, Akdis CA. Novel immunotherapy vaccine development. Curr Opin Allergy Clin Immunol. 2014;14(6):557-563

10. Hylander T, Latif L, Petersson-Westin U, Cardell LO. Intralymphatic allergen-specific immunotherapy: an effective and safe alternative treatment route for pollen-induced allergic rhinitis. J Allergy Clin Immunol. 2013;131 (2):412-420

11. Hartl D, et al. Quantitative and functional impairment of pulmonary CD4+CD25hi regulatory T cells in pediatric asthma. J Allergy Clin Immunol. 2007;119 (5):1258-1266.

12. Noval Rivas M, et al. Regulatory T cell reprogramming toward a Th2-cell-like lineage impairs oral tolerance and promotes food allergy. Immunity. 2015;42 (3):512-523.

13. Thorburn AN, Hansbro PM. Harnessing regulatory T cells to suppress asthma: from potential to therapy. Am J Respir Cell Mol Biol. 2010;43 (5):511-519.

14. Hankin CS, Cox L, Bronstone A, Wang Z. Allergy immunotherapy: reduced health care costs in adults and children with allergic rhinitis. J Allergy Clin Immunol. 2013;131 (4):1084-1091.

15. Hankin CS, et al. Allergen immunotherapy and health care cost benefits for children with allergic rhinitis: a large-scale, retrospective, matched cohort study. Ann Allergy Asthma Immunol. 2010;104 (1):79-85.

16. Al-Ramli W, et al. T (H)17-associated cytokines (IL-17A and IL-17F) in severe asthma. J Allergy Clin Immunol. 2009;123 (5):1185-1187.

17. Raundhal M, et al. High IFN- $\gamma$ and low SLPI mark severe asthma in mice and humans. J Clin Invest. $2015 ; 125$ (8):3037-3050.

18. Essilfie AT, et al. Macrolide therapy suppresses key features of experimental steroid-sensitive and steroid-insensitive asthma. Thorax. 2015;70 (5):458-467.

19. Wang W, Li JJ, Foster PS, Hansbro PM, Yang M. Potential therapeutic targets for steroid-resistant asthma. Curr Drug Targets. 2010;11 (8):957-970.

20. Yang M, Kumar RK, Hansbro PM, Foster PS. Emerging roles of pulmonary macrophages in driving the development of severe asthma. J Leukoc Biol. 2012;91 (4):557-569.

21. Steptoe RJ, Ritchie JM, Harrison LC. Transfer of hematopoietic stem cells encoding autoantigen prevents autoimmune diabetes. J Clin Invest. 2003;111 (9):1357-1363.

22. Steptoe RJ, Ritchie JM, Jones LK, Harrison LC. Autoimmune diabetes is suppressed by transfer of proinsulin-encoding Gr-1+ myeloid progenitor cells that differentiate in vivo into resting dendritic cells. Diabetes. 2005;54 (2):434-442.

23. Steptoe RJ, Ritchie JM, Wilson NS, Villadangos JA, Lew AM, Harrison LC. Cognate CD4+ help elicited by resting dendritic cells does not impair the induction of peripheral tolerance in CD8+ T cells. J Immunol. 2007;178 (4):2094-2103

24. Kenna TJ, Thomas R, Steptoe RJ. Steady-state dendritic cells expressing cognate antigen terminate memory CD8+ T-cell responses. Blood. 2008;111 (4):2091-2100.

25. Kenna TJ, et al. Targeting antigen to diverse APCs inactivates memory CD8+ T cells without eliciting tissue-destructive effector function. J Immunol. 2010;184 (2):598-606.

26. Nasreen M, Waldie TM, Dixon CM, Steptoe RJ. Steady-state antigen-expressing dendritic cells terminate CD4+ memory T-cell responses. Eur J Immunol. 2010;40 (7):2016-2025.

27. Coleman MA, et al. Tolerance induction with gene-modified stem cells and immune-preserving conditioning in primed mice: restricting antigen to differentiated antigen-presenting cells permits efficacy. Blood. 2013;121 (6):1049-1058.

28. Coleman MA, Steptoe RJ. Induction of antigen-specific tolerance through hematopoietic stem cell-mediated gene therapy: the future for therapy of autoimmune disease? Autoimmun Rev. 2012;12 (2):195-203.

29. Pape KA, Merica R, Mondino A, Khoruts A, Jenkins MK. Direct evidence that functionally impaired CD4+ T cells persist in vivo following induction of peripheral tolerance. J Immunol. 1998;160 (10):4719-4729.

30. Vendrame F, et al. Recurrence of type 1 diabetes after simultaneous pancreas-kidney transplantation, despite immunosuppression, is associated with autoantibodies and pathogenic autoreactive CD4 T-cells. Diabetes. 2010;59 (4):947-957.

31. Coleman MA, et al. Antigen-encoding bone marrow terminates islet-directed memory CD8+ T-cell responses to alleviate islet transplant rejection. Diabetes. 2016;65 (5):1328-1340.

32. Choi S, Schwartz RH. Molecular mechanisms for adaptive tolerance and other T cell anergy models. Semin Immunol. 2007;19 (3):140-152.

33. Wherry EJ. T cell exhaustion. Nat Immunol. 2011;12(6):492-499.

34. Ross EM, Bourges D, Hogan TV, Gleeson PA, van Driel IR. Helios defines T cells being driven to tolerance in the periphery and thymus. Eur J Immunol. 2014;44 (7):2048-2058.

35. Crawford A, et al. Molecular and transcriptional basis of $\mathrm{CD}^{+} \mathrm{T}$ cell dysfunction during chronic infection. Immunity. 2014;40 (2):289-302

36. McNally A, McNally M, Galea R, Thomas R, Steptoe RJ. Immunogenic, but not steady-state, antigen presentation permits regulatory T-cells to control CD8+ T-cell effector differentiation by IL-2 modulation. PLoS One. 2014;9 (1):e85455.

37. Erle DJ, Sheppard D. The cell biology of asthma. J Cell Biol. 2014;205(5):621-631.

38. Hansbro PM, Kaiko GE, Foster PS. Cytokine/anti-cytokine therapy - novel treatments for asthma? Br J Pharmacol. 2011;163 (1):81-95.

39. Kuperman DA, Huang X, Nguyenvu L, Hölscher C, Brombacher F, Erle DJ. IL-4 receptor signaling in Clara cells is required for 
allergen-induced mucus production. J Immunol. 2005;175 (6):3746-3752.

40. Doan T, McNally A, Thomas R, Steptoe RJ. Steady-state dendritic cells continuously inactivate T cells that escape thymic negative selection. Immunol Cell Biol. 2009;87(8):615-622.

41. Werner-Klein M, Dresch C, Marconi P, Brocker T. Transcriptional targeting of B cells for induction of peripheral CD8 T cell tolerance. J Immunol. 2007;178 (12):7738-7746.

42. Probst HC, Lagnel J, Kollias G, van den Broek M. Inducible transgenic mice reveal resting dendritic cells as potent inducers of CD8+ T cell tolerance. Immunity. 2003;18 (5):713-720.

43. Baranyi U, et al. Persistent molecular microchimerism induces long-term tolerance towards a clinically relevant respiratory allergen. Clin Exp Allergy. 2012;42 (8):1282-1292.

44. Hartley SB, Crosbie J, Brink R, Kantor AB, Basten A, Goodnow CC. Elimination from peripheral lymphoid tissues of self-reactive B lymphocytes recognizing membrane-bound antigens. Nature. 1991;353 (6346):765-769.

45. Goodnow CC, et al. Altered immunoglobulin expression and functional silencing of self-reactive B lymphocytes in transgenic mice. Nature. 1988;334(6184):676-682.

46. Baranyi U, et al. Tolerization of a type I allergic immune response through transplantation of genetically modified hematopoietic stem cells. J Immunol. 2008;180 (12):8168-8175.

47. Taylor JJ, et al. Deletion and anergy of polyclonal B cells specific for ubiquitous membrane-bound self-antigen. $J$ Exp Med. 2012;209 (11):2065-2077.

48. Baranyi U, et al. Cell Therapy for Prophylactic Tolerance in Immunoglobulin E-mediated Allergy. EBioMedicine 2016;7:230-239.

49. Bracy JL, Iacomini J. Induction of B-cell tolerance by retroviral gene therapy. Blood. 2000;96 (9):3008-3015.

50. Chung JY, et al. Gene therapy delivery of myelin oligodendrocyte glycoprotein (MOG) via hematopoietic stem cell transfer induces MOG-specific B cell deletion. J Immunol. 2014;192 (6):2593-2601.

51. Luger EO, Wegmann M, Achatz G, Worm M, Renz H, Radbruch A. Allergy for a lifetime? Allergol Int. 2010;59(1):1-8.

52. Niggemann B, et al. Five-year follow-up on the PAT study: specific immunotherapy and long-term prevention of asthma in children. Allergy. 2006;61 (7):855-859.

53. Jacobsen L, et al. Specific immunotherapy has long-term preventive effect of seasonal and perennial asthma: 10-year follow-up on the PAT study. Allergy. 2007;62 (8):943-948.

54. Kiel MA, Röder E, Gerth van Wijk R, Al MJ, Hop WC, Rutten-van Mölken MP. Real-life compliance and persistence among users of subcutaneous and sublingual allergen immunotherapy. J Allergy Clin Immunol. 2013;132 (2):353-60.e2.

55. Barnden MJ, Allison J, Heath WR, Carbone FR. Defective TCR expression in transgenic mice constructed using cDNA-based alpha- and beta-chain genes under the control of heterologous regulatory elements. Immunol Cell Biol. 1998;76 (1):34-40.

56. Murphy KM, Heimberger AB, Loh DY. Induction by antigen of intrathymic apoptosis of CD4+CD8+TCRlo thymocytes in vivo. Science. 1990;250 (4988):1720-1723.

57. Markey KA, et al. Conventional dendritic cells are the critical donor APC presenting alloantigen after experimental bone marrow transplantation. Blood. 2009;113 (22):5644-5649.

58. Horvat JC, et al. Neonatal chlamydial infection induces mixed T-cell responses that drive allergic airway disease. Am J Respir Crit Care Med. 2007;176 (6):556-564.

59. Starkey MR, et al. Constitutive production of IL-13 promotes early-life Chlamydia respiratory infection and allergic airway disease. Mucosal Immunol. 2013;6(3):569-579. 